\title{
The speed of a random front for stochastic reaction-diffusion equations with strong noise
}

\author{
Carl Mueller* Leonid Mytnik ${ }^{\dagger} \quad$ Lenya Ryzhik ${ }^{\ddagger}$
}

March 12, 2019

\begin{abstract}
We study the asymptotic speed of a random front for solutions $u_{t}(x)$ to stochastic reactiondiffusion equations of the form

$$
\partial_{t} u=\frac{1}{2} \partial_{x}^{2} u+f(u)+\sigma \sqrt{u(1-u)} \dot{W}(t, x), t \geq 0, x \in \mathbb{R},
$$

arising in population genetics. Here, $f$ is a continuous function with $f(0)=f(1)=0$, and such that $|f(u)| \leq K|u(1-u)|^{\gamma}$ with $\gamma \geq 1 / 2$, and $\dot{W}(t, x)$ is a space-time Gaussian white noise. We assume that the initial condition $u_{0}(x)$ satisfies $0 \leq u_{0}(x) \leq 1$ for all $x \in \mathbb{R}, u_{0}(x)=1$ for $x<L_{0}$ and $u_{0}(x)=0$ for $x>R_{0}$. We show that when $\sigma>0$, for each $t>0$ there exist $R\left(u_{t}\right)<+\infty$ and $L\left(u_{t}\right)<-\infty$ such that $u_{t}(x)=0$ for $x>R\left(u_{t}\right)$ and $u_{t}(x)=1$ for $x<L\left(u_{t}\right)$ even if $f$ is not Lipschitz. We also show that for all $\sigma>0$ there exists a finite deterministic speed $V(\sigma) \in \mathbb{R}$ so that $R\left(u_{t}\right) / t \rightarrow V(\sigma)$ as $t \rightarrow+\infty$, almost surely. This is in dramatic contrast with the deterministic case $\sigma=0$ for nonlinearities of the type $f(u)=u^{m}(1-u)$ with $0<m<1$ when solutions converge to 1 uniformly on $\mathbb{R}$ as $t \rightarrow+\infty$. Finally, we prove that when $\gamma>1 / 2$ there exists $c_{f} \in \mathbb{R}$, so that $\sigma^{2} V(\sigma) \rightarrow c_{f}$ as $\sigma \rightarrow+\infty$ and give a characterization of $c_{f}$. The last result complements a lower bound obtained by Conlon and Doering CD05] for the special case of $f(u)=u(1-u)$ where a duality argument is available.
\end{abstract}

\section{Introduction}

Reaction-diffusion equations of the form

$$
\partial_{t} u=\frac{1}{2} \partial_{x}^{2} u+f(u)
$$

with $f(0)=f(1)=0$, are often used to model biological invasions and other spreading phenomena, with one steady state, say, $u \equiv 1$ invading another, $u \equiv 0$, or vice versa. Under very mild assumptions on $f(u)$, such as, for instance, that $f(u)$ is Lipschitz on $[0,1]$ and either $f(u)>0$ for $u \in(0,1)$, or there exists $\theta \in(0,1)$ so that $f(u) \leq 0$ for $u \in(0, \theta)$ and $f(u)>0$ for $u \in(\theta, 1)$, such equations admit traveling wave solutions of the form $u_{t}(x)=U(x-c t)$ such that

$$
-c U^{\prime}=\frac{1}{2} U^{\prime \prime}+f(U), U(-\infty)=1, U(+\infty)=0
$$

\footnotetext{
${ }^{*}$ Department of Mathematics, University of Rochester, Rochester, NY, USA 14627; carl.e.mueller@rochester.edu

${ }^{\dagger}$ Faculty of Industrial Engineering and Management, Technion, Technion City, Haifa 3200003, Israel; leonid@ie.technion.ac.il

${ }^{\ddagger}$ Department of Mathematics, Stanford University, Stanford CA 94305, USA; ryzhik@math.stanford.edu
} 
Note that, in the probabilistic spirit of the present paper, the subscript $t$ denotes the time dependence of the function $u_{t}(x)$ rather than a time derivative, common to the PDE literature. It is easy to see that

$$
c \int_{\mathbb{R}}\left|U^{\prime}(x)\right|^{2} d x=\int_{0}^{1} f(z) d z,
$$

thus $c$ has the same sign as

$$
I[f]:=\int_{0}^{1} f(u) d u
$$

so that if $I[f]>0$ then the steady state $u \equiv 1$ is more stable, and invades the "less stable" steady state $u \equiv 0$, and if $I[f]<0$ then the opposite happens, while if $I[f]=0$ then (1.1) has a timeindependent solution. It is also well-known that traveling wave solutions to (1.1) determine the spreading speed for the solutions of the Cauchy problem. More precisely, let $u_{t}(x)$ be the solution to (1.1) with an initial condition $u_{0}(x)$ such that $0 \leq u_{0}(x) \leq 1$ for all $x \in \mathbb{R}$, and there exist $L_{0} \leq R_{0}$ so that $u_{0}(x)=1$ for $x<L_{0}$ and $u_{0}(x)=0$ for $x>R_{0}$. There exists a function $m(t)$ such that

$$
\left|m(t)-c_{*} t\right|=o(t) \text { as } t \rightarrow+\infty
$$

so that

$$
\left|u_{t}(x+m(t))-U_{c_{*}}(x)\right|=o(1) \text { as } t \rightarrow+\infty .
$$

Here, depending on the nature of the nonlinearity $f(u)$, the spreading speed $c_{*}$ may be either the speed of the unique traveling wave, or the minimal speed of a traveling wave if traveling waves are not unique. The latter happens for the class of the Fisher-KPP nonlinearities, such that $f$ is Lipschitz, $f(0)=f(1)=0, f(u)>0$ for all $u \in(0,1)$, and $f(u) \leq f^{\prime}(0) u$ for all $u \in[0,1]$. In that case, we have

$$
c_{*}=\sqrt{2 f^{\prime}(0)} .
$$

Much more precise results than (1.5)-(1.6) on the convergence of the solutions to the Cauchy problem to traveling waves are available, and we refer to the classical papers [AW78, Bra78, Bra83] for the basic results, and to [NRR18, Rob13] and references therein for more recent developments. We also point out the relation

$$
c_{*}=\lim _{t \rightarrow+\infty} \int_{\mathbb{R}} f\left(u_{t}(x)\right) d x=\int_{\mathbb{R}} f\left(U_{c_{*}}(x)\right) d x,
$$

that can be obtained simply by integrating (1.1) and (1.2) in space.

Note that if $f^{\prime}(0)$ blows up, then the speed of propagation may also tend to infinity, as can be seen from (1.7). For Hölder nonlinearities such that $f(u) \sim u^{p}$ with $p \in(0,1)$, it was shown in AE86. that solutions become instantaneously strictly positive everywhere: $u(t, x) \geq c t^{1 /(1-p)}$ for $t \ll 1$. In particular, if we approximate such nonlinearity by a sequence of Lipschitz nonlinearities $f_{n}$, then the corresponding spreading speeds $c_{*}^{(n)}$ blow up as $n \rightarrow+\infty$.

\section{Reaction-diffusion equations with noise}

The physical and biological systems modeled by reaction-diffusion equations are often subject to noise. In this paper, we study solutions $u_{t}(x)$, to the stochastic reaction-diffusion equations of the form

$$
\partial_{t} u=\frac{1}{2} \partial_{x}^{2} u+f(u)+\sigma \sqrt{u(1-u)} \dot{W}(t, x)
$$

where $\dot{W}(t, x)$ is a space-time Gaussian white noise, and $\sigma>0$ measures its strength. Our interest is in the effect of the noise term on the spreading speed. Since traveling waves will no longer maintain 
a fixed shape due to the noise, we will refer instead to the speed of the random front, which is defined below.

Let us give an motivation for the noise term in (1.9) similar to that given by Fisher in his pioneering work [Fis37]. See also Shi88. Imagine that two populations, type A and type B, move in a Brownian way along $\mathbb{R}$, and let $u_{t}(x)$ is the proportion of the population of type $\mathrm{A}$ at time $t$ at position $x$. When an individual of type A meets an individual of type $\mathrm{B}$, it can be converted into type $\mathrm{B}$, and vice versa, and the outcome is partially random. The function $f(u)$ in (1.9) describes the deterministic evolution of the population of type A, due to these interactions, and it is natural to assume that $f(0)=f(1)=0$ since there are no interactions when one type is absent. The random term in (1.9) accounts for the stochastic aspect of the interactions. We assume that for each such meeting we have a mean-zero random variable affecting the outcome, and these random variables are i.i.d. By the central limit theorem, the sum of such variables would be approximately Gaussian. The independence of the variables means that the random input should be independent for different values of $t$ and $x$, giving rise to the space-time noise $\dot{W}(t, x)$. The rate of such meetings at a given site $x$ and time $t$ would be proportional to $u_{t}(x)\left(1-u_{t}(x)\right)$, which is the variance of the noise at $(t, x)$. Thus we should multiply the white noise $\dot{W}(t, x)$ by the standard deviation $\sqrt{u_{t}\left(1-u_{t}\right)}$. This leads to the noise term in (1.9).

As we have mentioned, we are interested in the long time speed of a random front for the solutions to (1.9). To this end, we define the left and the right edge of the solution as follows. Given a function $h(x)$ such that $0 \leq h(x) \leq 1$ for all $x \in \mathbb{R}$, with $h(x) \rightarrow 1$ as $x \rightarrow-\infty$ and $h(x) \rightarrow 0$ as $x \rightarrow+\infty$, we set

$$
\begin{aligned}
& L(h)=\inf \{x \in \mathbb{R}: h(x)<1\} \\
& R(h)=\sup \{x \in \mathbb{R}: h(x)>0\} .
\end{aligned}
$$

In the absence of the noise, when $\sigma=0$, and for Lipschitz nonlinearities $f(u)$, we have $L\left(u_{t}\right)=-\infty$ and $R\left(u_{t}\right)=+\infty$ for all $t>0$. This, however, is not necessarily the case in the presence of the noise. In order to make this claim precise, we assume that

$$
f \text { is continuous on }[0,1] \text { and there exists } K_{f}>0 \text { such that } f(u) \leq K_{f} \sqrt{|u(1-u)|} .
$$

As for the initial condition $u_{0}(x)$, we will assume that

$$
0 \leq u_{0}(x) \leq 1 \text { for all } x \in \mathbb{R} \text {, and both } L\left(u_{0}\right) \text { and } R\left(u_{0}\right) \text { are finite. }
$$

We will denote by $\mathcal{C}_{I}$ the set of continuous functions satisfying (1.12). In addition $\widehat{\mathcal{B}}_{I}$ will denote the space of functions on $\mathbb{R}$ taking values in $[0,1]$ and $\widehat{\mathcal{C}_{I}}$ will denote the space of continuous functions on $\mathbb{R}$ taking values in $[0,1]$.

We say that $u_{t}$ has a speed $V(\sigma)$ if the following limit exists:

$$
V(\sigma)=\lim _{t \rightarrow \infty} \frac{R\left(u_{t}\right)}{t} .
$$

We prove the following theorem in Section 2 ,

Theorem 1.1. Let $f(u)$ satisfy (1.11) and $u_{0}(x)$ be as in (1.12), then (1.9) with an initial condition $u_{0}(x)$ has a solution $u_{t}(x)$ taking values in $\widehat{\mathcal{C}}_{I}$ for $t>0$. The solution is unique in law. Moreover, $L\left(u_{t}\right)$ and $R\left(u_{t}\right)$ are almost surely finite for all $t \geq 0$ and the solution has a speed $V(\sigma) \in \mathbb{R}$.

We see that the noise has a very strong slowdown effect: $V(\sigma)$ is finite for all $\sigma>0$ even if $f(u)$ is Hölder with an exponent $m \geq 1 / 2$, and not Lipschitz, such as, for instance $f(u)=u^{m}(1-u)$, for which, as we have mentioned, the speed of the front is infinite when $\sigma=0$. 
Most of the papers dealing with (1.9), such as Mueller and Sowers [MS95] have treated the FisherKPP nonlinearity $f(u)=u(1-u)$, and small noise, where $\sigma$ is close to 0. Mueller, Mytnik, and Quastel MMQ11] studied the behavior of $V(\sigma)$ as $\sigma \downarrow 0$ and verified some conjectures of Brunet and Derrida [BD97] and [BD00]. Less attention has been devoted to $V(\sigma)$ for large or intermediate values of $\sigma$, but Conlon and Doering [CD05] proved that for $f(u)=u(1-u)$ there exists an asymptotic velocity $V(\sigma)>0$ for solutions $u$ to (1.9) for all $\sigma>0$, and that

$$
\liminf _{\sigma \rightarrow \infty} \sigma^{2} V(\sigma) \geq 1
$$

Note that (1.13) differs from (1.7) in [CD05] because the diffusivity in that paper is taken to be 1 rather than $1 / 2$ as chosen here. To formulate our main result, we note that a rescaling of (1.9), discussed in Section 2 allows us to move the noise coefficient into the nonlinearity, and obtain the rescaled equation

$$
\partial_{t} v=\frac{1}{2} \partial_{x}^{2} v+\sigma^{-4} f(v)+\sqrt{v(1-v)} \dot{W}(t, x) .
$$

Here $v$ is a rescaling of $u$ which we specify later. Later we will use the results of Tribe [Tri95, and Mueller and Tribe [MT97] for (1.14) with $f=0$, a version of a continuous voter model, or a stepping stone model in population genetics:

$$
\partial_{t} w=\frac{1}{2} \partial_{x}^{2} w+\sqrt{w(1-w)} \dot{W}(t, x) .
$$

By Theorem 1 of [MT97], we know that $w_{t}\left(x-R\left(w_{t}\right)\right)$ converges weakly to a stationary distribution as $t \rightarrow \infty$. We denote the expectation with respect to the stationary distribution of $w$ by $\mathbb{E}_{w, s t}$, where "st" is an abbreviation for "stationary". For the next theorem we need an assumption on $f$ which is slightly stronger than (1.11): we assume

$$
f \text { is continuous on }[0,1] \text { and there exists } \widetilde{K}_{f}>0 \text { s.t. } f(u) \leq \widetilde{K}_{f}|u(1-u)|^{\gamma} \text { for some } \gamma \in(1 / 2,1] \text {. }
$$

Theorem 1.2. Suppose that $u_{0}$ satisfies (1.12) and $f$ satisfies (1.16). Then we have, almost surely,

$$
\lim _{\sigma \rightarrow \infty} \sigma^{2} V(\sigma)=c_{f}
$$

where

$$
c_{f} \equiv \mathbb{E}_{w, s t}\left[\int_{\mathbb{R}} f(w(x)) d x\right]
$$

and

$$
\left|c_{f}\right|<\infty
$$

Note that Lemma 2.1 of [Tri95] shows that

$$
\lim _{t \rightarrow \infty} \mathbb{E}_{w}\left[\int_{\mathbb{R}} w_{t}(x)\left(1-w_{t}(x)\right) d x\right]=1
$$

This immediately implies that $\left|c_{f}\right|<\infty$ for $f$ satisfying (1.16) with $\gamma=1$. In particular, as a consequence of Theorem 1.2, we get that for the Fisher-KPP nonlinearity $f(u)=u(1-u)$, we have

$$
\lim _{\sigma \rightarrow \infty} \sigma^{2} V(\sigma)=1
$$

giving a matching upper bound to the lower bound (1.13) of Conlon and Doering in CD05, after adjusting for the different diffusivities adopted in the present paper and in CD05]. For the general $f$ satisfying (1.16), we show that (1.19) holds in Lemma 3.4. 
We also see the slowdown due to strong noise in Theorem 1.2 even for Lipschitz nonlinearities. The large noise asymptotics in (1.17) corresponds to the speed of the front for solutions of (1.14) that is $V^{(v)}(\sigma) \sim c_{f} / \sigma^{4}$. However, solutions of the corresponding equation without the noise

$$
\partial_{t} v=\frac{1}{2} \partial_{x}^{2} v+\sigma^{-4} f(v)
$$

spread with the speed $\bar{V}(\sigma)=c_{*} / \sigma^{2}$, where $c_{*}$ is the speed of the traveling wave for (1.21) with $\sigma=1$, so that $V^{(v)}(\sigma) \ll \bar{V}(\sigma)$ for $\sigma \gg 1$, and the noise slows down the propagation.

Let us also point out that expression (1.17)-(1.18) for the front speed $V(\sigma)$ is a direct analog of (1.8) except now the role of the traveling wave is played by the invariant measure of $w_{t}(x)$. One may conjecture that instead of the convergence to a traveling wave in shape, as in (1.6) that happens in the deterministic case, here, in the limit $\sigma \rightarrow+\infty$, the law of $u_{t}(x)$ after rescaling converges, as $t \rightarrow+\infty$, in the frame moving with the speed $V(\sigma)$, to the invariant distribution of $w_{t}(x)$.

Another interesting observation is that the noise, despite its symmetry with respect to $u=0$ and $u=1$ can change the direction of the invasion. One may construct a nonlinearity $f$ such that $I(f)$ given by (1.4) has a different sign than $c_{f}$, meaning that that the speed of propagation for $\sigma=0$, in the absence of the noise, may have a different sign than $V(\sigma)$ for large $\sigma \gg 1$, changing the direction of the invasion, because of the noise.

The paper is organized as follows. The proof of Theorem 1.1 is in Section 2. Section 3 contains some auxiliary results on solutions to (1.15). They are used later in the proof of Theorem 1.2, presented in Sections 4 for the upper bound, and in Section 5 for the matching lower bound on the speed $V(\sigma)$ for $\sigma \gg 1$.

Acknowledgement. The work of LM and LR was supported by a US-Israel BSF grant. LR was supported by NSF grant DMS-1613603 and ONR grant N00014-17-1-2145, and CM was supported by a Simons Grant.

\section{The proof of Theorem 1.1}

In this section, we prove Theorem 1.1. Existence of a solution to (1.9) follows by a rather standard argument. To prove the uniqueness, we use Girsanov's theorem. In order to be able to apply this theorem, we need to have an a priori bound showing that for any solutions to (1.9) taking values

in $\widehat{\mathcal{B}}_{I}$ for all $t \geq 0$ with $R\left(u_{0}\right)<+\infty, L\left(u_{0}\right)>-\infty$, we have $-\infty<L\left(u_{t}\right)<R\left(u_{t}\right)<+\infty$ for all $t \geq 0$, almost surely.

\section{$2.1 \quad$ Existence of a solution}

We first show that (1.9) has a mild solution. The notion of a mild solution to (1.9) follows the standard definition, see Walsh Wal86]. We interpret (1.9) as a shorthand for the mild form,

$$
\begin{aligned}
u_{t}(x)= & \int_{\mathbb{R}} G_{t}(x-y) u_{0}(y) d y+\int_{0}^{t} \int_{\mathbb{R}} G_{t-s}(x-y) f\left(u_{s}(y)\right) d y d s \\
& +\int_{0}^{t} \int_{\mathbb{R}} G_{t-s}(x-y) \sqrt{u_{s}(y)\left(1-u_{s}(y)\right)} W(d y d s),
\end{aligned}
$$

where $u_{0}(x)$ is the given initial condition. Here,

$$
G_{t}(x)=(2 \pi t)^{-1 / 2} \exp \left(-x^{2} /(2 t)\right),
$$


is the fundamental solution of the heat equation

$$
\partial_{t} G=\frac{1}{2} \partial_{x}^{2} G
$$

In what follows, with some abuse of notation $\left\{G_{t}, t \geq 0\right\}$ will also denote the corresponding semigroup, that is,

$$
G_{t} \phi(x)=\int_{\mathbb{R}} G_{t}(x-y) \phi(y), d y, t>0,
$$

for any function $\phi$ for which the above integral is well-defined.

Almost sure existence and uniqueness of mild solutions to SPDEs of the form

$$
\partial_{t} u=\frac{1}{2} \partial_{x}^{2} u+f(u)+a(u) \dot{W}(t, x)
$$

is standard [Wal86] when the coefficients are Lipschitz continuous functions of $u$. Because in our case $f(u)$ may be not Lipschitz, and $a(u)=\sqrt{u(1-u)}$ is not Lipschitz, one needs to be slightly more careful. Solutions to (1.9) are constructed as follows. Let the initial condition $u_{0}$ satisfy (1.12). We approximate $f(u)$ and $a(u)$ by Lipschitz functions $f_{n}(u)$ and $a_{n}(u)$ such that

$$
f_{n}(0)=f_{n}(1)=a_{n}(0)=a_{n}(1)=0,
$$

and construct the corresponding solutions $u_{t}^{n}(x)$ using the standard theory. The comparison principle implies that $u_{t}^{n}(x)$ take values in [0,1], see [Shi94] and [Mue91. The proof of Theorem 2.6 of [Shi94, on pp. 436-437 of that paper, shows that the sequence $u_{t}^{n}(x)$ is tight. Passing to the limit $n \rightarrow+\infty$ we obtain a mild solution $u_{t}(x)$ to (1.9) taking values in $[0,1]$. This proves existence of a solution.

\subsection{Uniqueness via the Girsanov theorem}

In order to prove uniqueness in law of the solution to (1.9), we will use a version of the Girsanov theorem that will allow us to compare the laws of the solution $u_{t}(x)$ to (1.9) and $w_{t}(x)$, the solution to (1.15), which corresponds to $f=0$ in (1.9), with the same initial condition $w_{0}(x)=u_{0}(x)$. Recall that we have set $\sigma=1$, including in (1.15). Let $\mathbb{P}_{t, u}$ be the measure induced on the canonical path space up to time $t$ by $u$, and $\mathbb{P}_{t, w}$ be the measure induced by $w$, also up to time $t$. We also define the corresponding expectations $\mathbb{E}_{t, u}$ and $\mathbb{E}_{t, w}$, and write $\mathbb{P}_{u}$ for $\mathbb{P}_{\infty, u}$, and likewise $\mathbb{P}_{w}$ for $\mathbb{P}_{\infty, w}$. We will not use the subscripts in the situations when it is clear which probability measure is used.

In [Daw78], Dawson gives a version of Girsanov's theorem which applies to $\mathbb{P}_{t, u}$ and $\mathbb{P}_{t, w}$. We will use its variant, Theorem IV.1.6 in [Per02]. In such theorems, the change of measure always involves an exponential term which must be a martingale. In our situation, let

$$
Z_{t}=\int_{0}^{t} \int_{\mathbb{R}} \frac{f\left(w_{s}(x)\right)}{\sqrt{w_{s}(x)\left(1-w_{s}(x)\right)}} W(d x, d s)-\frac{1}{2} \int_{0}^{t} \int_{\mathbb{R}} \frac{f\left(w_{s}(x)\right)^{2}}{w_{s}\left((x) 1-w_{s}(x)\right)} d x d s .
$$

Here, and elsewhere we adopt the convention in the integrands that

$$
\frac{f(u)}{\sqrt{u(1-u)}}=0 \text { if } u=0 \text { or } u=1
$$

Then Girsanov's theorem for stochastic PDE [Daw78, Per02] says that

$$
\frac{d \mathbb{P}_{t, u}}{d \mathbb{P}_{t, w}}=e^{Z_{t}}
$$


as long as

$$
\int_{0}^{t} \int_{\mathbb{R}} \frac{f\left(u_{s}(x)\right)^{2}}{u_{s}(x)\left(1-u_{s}(x)\right)} d x d s<+\infty, \quad \mathbb{P}_{u^{-} \text {almost surely. }}
$$

In particular, if (2.6) holds then (2.5) implies immediately that the solution to (1.9) is unique in law. For the moment, as we do not have any information on the support of $f\left(u_{s}(x)\right)$, we can not conclude that (2.6) holds. The bulk of the rest of this section is to show that (2.6) holds for any solution to (1.9) taking values in $\widehat{\mathcal{B}}_{I}$ for all $t \geq 0$ and such that $R\left(u_{0}\right)<+\infty$ and $L\left(u_{0}\right)>-\infty$.

First, we make a much simpler observation that allows us to use Girsanov's theorem to eliminate the drift on a finite interval. Fix and arbitrary $b>0$ and let $v^{b}$ denote a solution to a modified version of (1.9), with the nonlinearity set to zero on the interval $[-10 b, 10 b]$ :

$$
\partial_{t} v_{t}^{b}(x)=\frac{1}{2} \partial_{x}^{2} v_{t}^{b}(x)+f\left(v_{t}^{b}(x)\right) \mathbf{1}_{\{x \in(-\infty,-10 b) \cup(10 b, \infty)\}}+\sqrt{v_{t}^{b}(x)\left(1-v_{t}^{b}(x)\right)} \dot{W}(t, x) .
$$

We again write this equation in the mild form:

$$
v_{t}^{b}(x)=G_{t} v_{0}^{b}(x)+\int_{0}^{t} \int_{\mathbb{R}} G_{t-s}(x-z) f\left(v_{s}^{b}(z)\right) \mathbf{1}_{\{z \in(-\infty,-10 b) \cup(10 b, \infty)\}} d z+N_{t}^{b}(x),
$$

where

$$
N_{t}^{b}(x)=\int_{0}^{t} \int_{\mathbb{R}} G_{t-s}(x-z) \sqrt{v_{s}^{b}(z)\left(1-v_{s}^{b}(z)\right)} W(d s, d z) .
$$

Let $\mathbb{P}_{t, v^{b}}$ be the measure induced on the canonical path space up to time $t$ by $v^{b}$, with the corresponding expectation $\mathbb{E}_{t, v^{b}}$, and $\mathbb{P}_{\infty, v^{b}}$ be $\mathbb{P}_{v^{b}}$. Note that by (1.11) we have

$$
\int_{0}^{t} \int_{\mathbb{R}} \frac{f\left(u_{s}(x)\right)^{2} \mathbf{1}_{\{x \in(-10 b, 10 b)\}}}{u_{s}(x)\left(1-u_{s}(x)\right)} d x d s \leq 20 b K_{f}^{2} t<+\infty, \quad \mathbb{P}_{u^{-}} \text {-almost surely. }
$$

Thus we can use Girsanov's theorem for stochastic PDE [Daw78, Per02] to get

$$
\frac{d \mathbb{P}_{t, u}}{d \mathbb{P}_{t, v^{b}}}=e^{Z_{t}^{b}}
$$

where

$$
Z_{t}^{b}=\int_{0}^{t} \int_{\mathbb{R}} \frac{f\left(v_{s}^{b}(x)\right) \mathbf{1}_{\{x \in(-10 b, 10 b)\}}}{\sqrt{v_{s}^{b}(x)\left(1-v_{s}^{b}(x)\right)}} W(d x, d s)-\frac{1}{2} \int_{0}^{t} \int_{\mathbb{R}} \frac{f\left(v_{s}^{b}(x)\right)^{2} \mathbf{1}_{\{x \in(-10 b, 10 b)\}}}{v_{s}^{b}\left((x) 1-v_{s}^{b}(x)\right)} d x d s .
$$

\subsection{A bound on the front speed}

The next step is to get the following bound on the speed of the front of $u$.

Lemma 2.1. Let $u_{t}(x)$ be a solution to (1.9) taking values in $\widehat{\mathcal{B}}_{I}$ for all $t \geq 0$ such that the initial condition $u_{0}(x)$ satisfies (1.12) with $R\left(u_{0}\right) \leq 0$. Then, for all $T>0$, both $\sup _{t \leq T} R\left(u_{t}\right)$ and $\sup _{t \leq T} L\left(u_{t}\right)$ are almost surely finite. Moreover, for all $T \geq 0$ there exists $C_{T}>0$ so that for all $b \geq 4 \sqrt{T}\left(T\|f\|_{\infty} \vee 1\right)$ we have

$$
\mathbb{P}\left(\sup _{0 \leq t \leq T}\left|R\left(u_{t}\right)-R_{0}\right|>b\right)+\mathbb{P}\left(\sup _{0 \leq t \leq T}\left|L\left(u_{t}\right)-L_{0}\right|>b\right) \leq C_{T} \exp \left(-\frac{b^{2}}{100 T}\right) .
$$

An immediate consequence is

Corollary 2.2. We have, for each $T \geq 0$ :

$$
\mathbb{E}\left[\sup _{0 \leq t \leq T}\left|R\left(u_{t}\right)-L\left(u_{t}\right)\right|\right]<+\infty .
$$

In other words, any solution to (1.9) has an interface that has a finite length almost surely. 


\section{Bounds on the martingale with the cut-off}

The proof of Lemma 2.1 relies on a priori bounds on the propagation of $v^{b}$, solution to (2.7). First, we need to control the modulus of continuity of the martingale $N_{t}^{b}(\cdot)$ defined in (2.9)).

Lemma 2.3. Let $v_{t}^{b}(x)$ be a solution to (2.7) taking values in $\widehat{\mathcal{B}}_{I}$ for all $t \geq 0$, such that the initial condition $v_{0}^{b}(x)$ satisfies (1.12) with $R\left(v_{0}^{b}\right) \leq 0$. Then, for all $p \geq 1$, there exists $C(p)>0$ so that for all $t \geq 0$, and $x, y \in[b / 2,9 b]$ we have

$$
\begin{aligned}
\mathbb{E}\left[\left|N_{t}^{b}(x)-N_{t}^{b}(y)\right|^{2 p}\right] & \leq C(p)\left(|x-y| \wedge t^{1 / 2}\right)^{p-1} t^{1 / 2} \\
& \times \int_{\mathbb{R}}\left(G_{t}(x-z)+G_{t}(y-z)\right)\left(v_{0}^{b}(z)+t\|f\|_{\infty} \mathbf{1}_{\{z \in(-\infty,-10 b) \cup(10 b, \infty)\}}\right) d z, \\
\mathbb{E}\left[\left|N_{t}^{b}(x)-N_{s}^{b}(x)\right|^{2 p}\right] & \leq C(p)|t-s|^{(p-1) / 2} t^{1 / 2} \\
& \times \int_{\mathbb{R}}\left(G_{t}(x-z)+G_{s}(x-z)\right)\left(v_{0}^{b}(z)+t\|f\|_{\infty} \mathbf{1}_{\{z \in(-\infty,-10 b) \cup(10 b, \infty)\}}\right) d z .
\end{aligned}
$$

Proof. The proof follows the lines of the proof of Lemma 3.1 in [Tri95]. We only verify (2.15). Note that

$$
\int_{0}^{t} \int_{\mathbb{R}}\left(G_{t-s}(x-z)-G_{t-s}(y-z)\right)^{2} d z d s \leq C\left(|x-y| \wedge t^{1 / 2}\right) \forall t>0, x, y \in \mathbb{R} .
$$

Burkholder's and Hölder's inequalities give

$$
\begin{aligned}
& \mathbb{E}\left[\left|N_{t}^{b}(x)-N_{t}^{b}(y)\right|^{2 p}\right] \leq C(p) \mathbb{E}\left[\left(\int_{0}^{t} \int_{\mathbb{R}}\left(G_{t-s}(x-z)-G_{t-s}(y-z)\right)^{2} v_{s}^{b}(z)\left(1-v_{s}^{b}(z)\right) d z d s\right)^{p}\right] \\
& \leq C(p)\left(|x-y| \wedge t^{1 / 2}\right)^{p-1} \mathbb{E}\left[\int_{0}^{t} \int_{\mathbb{R}}\left(G_{t-s}(x-z)-G_{t-s}(y-z)\right)^{2}\left(v_{s}^{b}(z)\left(1-v_{s}^{b}(z)\right)\right)^{p} d z d s\right] \\
& \leq C(p)\left(|x-y| \wedge t^{1 / 2}\right)^{p-1} \mathbb{E}\left[\int_{0}^{t} \int_{\mathbb{R}}\left(G_{t-s}(x-z)-G_{t-s}(y-z)\right)^{2} v_{s}^{b}(z) d z d s\right] \\
& \leq C(p)\left(|x-y| \wedge t^{1 / 2}\right)^{p-1} \mathbb{E}\left[\int_{0}^{t}(t-s)^{-1 / 2} \int_{\mathbb{R}}\left(G_{t-s}(x-z)+G_{t-s}(y-z)\right) v_{s}^{b}(z) d z d s\right] .
\end{aligned}
$$

We used the fact that $0 \leq v^{b} \leq 1$ in the third inequality above. Note that

$$
\begin{aligned}
\mathbb{E}\left[v_{s}^{b}(x)\right] & =G_{s} v_{0}^{b}(x)+\mathbb{E}\left[\int_{0}^{s} \int_{\mathbb{R}} G_{s-r}(x-z) f\left(v_{r}^{b}(z)\right) \mathbf{1}_{\{z \in(-\infty,-10 b) \cup(10 b, \infty)\}} d z d r\right] \\
& \left.\leq G_{s} v_{0}^{b}(x)+\|f\|_{\infty} \int_{0}^{s} \int_{\mathbb{R}} G_{s-r}(x-z) \mathbf{1}_{\{z \in(-\infty,-10 b) \cup(10 b, \infty)\}}\right) d z d r .
\end{aligned}
$$

We substitute this bound into the right side of (2.18) and use the semi-group property of $G_{t}$ to get

$$
\begin{aligned}
\mathbb{E}\left[\left|N_{t}^{b}(x)-N_{t}^{b}(y)\right|^{2 p}\right] \leq & C(p)\left(|x-y| \wedge t^{1 / 2}\right)^{p-1}\left\{\int _ { 0 } ^ { t } ( t - s ) ^ { - 1 / 2 } \left(\int_{\mathbb{R}}\left(G_{t}(x-z)+G_{t}(y-z)\right) v_{0}^{b}(z) d z\right.\right. \\
& \left.\left.\left.+\int_{0}^{s}\|f\|_{\infty} \int_{\mathbb{R}}\left(G_{t-r}(x-z)+G_{t-r}(y-z)\right) \mathbf{1}_{\{z \in(-\infty,-10 b) \cup(10 b, \infty)\}}\right) d z d r\right) d s\right\} \\
\leq & C(p)\left(|x-y| \wedge t^{1 / 2}\right)^{p-1} t^{1 / 2}\left(\int_{\mathbb{R}}\left(G_{t}(x-z)+G_{t}(y-z)\right) v_{0}^{b}(z) d z\right. \\
& \left.\left.+\|f\|_{\infty} \int_{0}^{t} \int_{\mathbb{R}}\left(G_{t-r}(x-z)+G_{t-r}(y-z)\right) \mathbf{1}_{\{z \in(-\infty,-10 b) \cup(10 b, \infty)\}}\right) d z d r\right) .
\end{aligned}
$$


Since $x, y \in(b / 2,9 b)$ and $z \geq 10 b$ we have

$$
\int_{z \geq 10 b} G_{r}(x-z) d z \leq \int_{z \geq 10 b} G_{t}(x-z) d z, \quad \forall x \in(b / 2,9 b), 0 \leq r \leq t,
$$

and thus we get

$$
\begin{aligned}
\mathbb{E}\left[\left|N_{t}^{b}(x)-N_{t}^{b}(y)\right|^{2 p}\right] & \leq C(p)\left(|x-y| \wedge|t-s|^{1 / 2}\right)^{p-1} \\
& \times t^{1 / 2} \int_{\mathbb{R}}\left(G_{t}(x-z)+G_{t}(y-z)\right)\left(v_{0}^{b}(z)+t\|f\|_{\infty} \mathbf{1}_{\{z \in(-\infty,-10 b) \cup(10 b, \infty)\}}\right) d z,
\end{aligned}
$$

which is (2.15). The proof of (2.16) $)$ goes along similar lines.

A corollary of Lemma 2.3 is a bound on the size of $N_{b}^{s}(x)$.

Lemma 2.4. Let $v_{t}^{b}(x)$ be a solution to 2.7), taking values in $\widehat{\mathcal{B}}_{I}$ for all $t \geq 0$, and the initial condition $v_{0}^{b}(x)$ satisfies (1.12) with $R\left(v_{0}^{b}\right) \leq 0$. Then, for all $t>0$, there exists $C$ such that

$$
\begin{aligned}
& \mathbb{P}\left(\left|N_{s}^{b}(x)\right| \geq \varepsilon \text { for some } x \in(b / 2,9 b), s \in[0, t]\right) \\
& \left.\quad \leq C \varepsilon^{-20}\left(t \vee t^{22}\right) \int_{\mathbb{R}} \int_{\mathbb{R}} G_{t}(x-z)\left(v_{0}^{b}(z)+t\|f\|_{\infty} \mathbf{1}_{\{z \in(-\infty,-10 b) \cup(10 b, \infty)\}}\right) d z \mathbf{1}_{\{x \in(b / 2,9 b)\}}\right) d x .
\end{aligned}
$$

Proof. The proof goes exactly as the second part of the proof of Lemma 3.1 in Tri95] (on p. 295) while taking $v_{0}^{b}(z)+t\|f\|_{\infty} \mathbf{1}_{\{z \in(-\infty,-10 b) \cup(10 b, \infty)\}}$ instead of $f$ and $(b / 2,9 b)$ instead of $(A, \infty)$ there.

\section{The support of the solution with a cut-off}

Now, we prove the following lemma.

Lemma 2.5. Let $v_{t}^{b}(x)$ be a solution to (2.7) taking values in $\widehat{\mathcal{B}}_{I}$ for all $t \geq 0$ such that the initial condition $v_{0}^{b}(x)$ satisfies (1.12) with $R\left(v_{0}^{b}\right) \leq 0$. Then, for all $t>0$ there exists $C_{t}>0$ so that for all $b \geq 4 \sqrt{t}\left(t\|f\|_{\infty} \vee 1\right)$ we have

$$
\mathbb{P}\left(\sup _{0 \leq s \leq t} \sup _{x \in[b, 2 b]} v_{s}^{b}(x)>0\right) \leq C\left(t,\|f\|_{\infty}\right) \exp \left(-\frac{b^{2}}{50 t}\right) .
$$

Proof. We will follow the proof of Proposition 3.2 in [Tri95]. Let us take a function $\psi \in L^{1}(\mathbb{R}) \cap C(\mathbb{R})$ such that $0 \leq \psi(x) \leq 1$ for all $x \in \mathbb{R}$ and $\{x: \psi(x)>0\}=(0, b)$, and set $\psi_{b}(x)=\psi(x-b)$. For simplicity of notation, we define

$$
\langle h, g\rangle=\int_{\mathbb{R}} h(x) g(x) d x
$$

for any functions $h, g$ such that the integral above exists.

Fix $t>0$ and let $\phi_{s}^{\lambda}(x), 0 \leq s \leq t, x \in \mathbb{R}$ be the unique non-negative bounded solution to the backward in time problem

$$
-\partial_{s} \phi_{s}^{\lambda}=\frac{1}{2} \Delta \phi_{s}^{\lambda}-\frac{1}{4}\left(\phi_{s}^{\lambda}\right)^{2}+\lambda \psi_{b}
$$

with the terminal condition $\phi_{t}^{\lambda}(x) \equiv 0$. A similar equation to (2.24) but with different function $\psi_{b}$ in the right side appears in the proof of Proposition 3.2 in [Tri95]. As $\psi_{b}(x) \geq 0$ for all $x \in \mathbb{R}$, the maximum principle implies existence of the solution to (2.24) and that $\phi_{s}^{\lambda}(x) \geq 0$ for all $0 \leq s \leq t$ and $x \in \mathbb{R}$. The maximum principle also implies that

$$
\phi_{s}^{\lambda}(x) \leq \lambda \int_{0}^{t-s} \int G_{r}(x-y) \psi_{b}(y) d y d r, \quad s \leq t,
$$


and thus $\phi_{s}^{\lambda}(x)$ is integrable for all $0 \leq s \leq t$. Next, note that the function

$$
\zeta_{t}(x)=\left\{\begin{array}{l}
\frac{\alpha}{(x-b)^{2}}, x<b, \\
\frac{\alpha}{(x-2 b)^{2}}, x>2 b,
\end{array}\right.
$$

satisfies, in the region $x<b$, where $\psi_{b}(x) \equiv 0$ :

$$
\partial_{t} \zeta-\frac{1}{2} \Delta \zeta+\frac{1}{4} \zeta^{2}-\lambda \psi_{b}=-\frac{1}{2} \frac{2 \cdot 3 \alpha}{(x-b)^{4}}+\frac{\alpha^{2}}{4(x-b)^{4}}=\frac{\alpha(\alpha-12)}{(x-b)^{4}} \geq 0
$$

provided that we take $\alpha \geq 12$. As $\zeta_{t}(x)=+\infty$ at $x=b$, the maximum principle implies that, for $\alpha$ sufficiently large, we have

$$
\phi_{s}^{\lambda}(x) \leq \frac{\alpha}{(b-x)^{2}}, \quad \text { for all } x<b, s \leq t, \text { and } \lambda>0 .
$$

Similarly, again for $\alpha$ large enough, we get

$$
\phi_{s}^{\lambda}(x) \leq \frac{\alpha}{(2 b-x)^{2}}, \text { for all } x>2 b, s \leq t, \text { and } \lambda>0 .
$$

Now, given any $b \geq 4 t^{1 / 2}$, we may use the fundamental solution for the heat equation on the halflines $x<b-t^{1 / 2}, x>2 b+t^{1 / 2}$ together with the upper bound in (2.25) on $\phi_{s}^{\lambda}(x)$ at $x=b-t^{1 / 2}$, and $x=2 b+t^{1 / 2}$ to conclude that there exists $\alpha_{1}>0$ such that

$$
\phi_{s}^{\lambda}(x) \leq \frac{\alpha_{1}}{t} \exp \left(-\frac{(b-x)^{2}}{20 t}\right), \quad \text { for all } b \geq 4 t^{1 / 2}, x<b-2 t^{1 / 2}, s \leq t, \text { and } \lambda>0,
$$

and

$$
\phi_{s}^{\lambda}(x) \leq \frac{\alpha_{1}}{t} \exp \left(-\frac{(2 b-x)^{2}}{20 t}\right), \text { for all } b \geq 4 t^{1 / 2}, x>2 b+2 t^{1 / 2}, s \leq t, \text { and } \lambda>0 .
$$

Next, by Itô's formula, we get, for any $0 \leq s \leq t$ :

$$
\begin{aligned}
\exp ( & \left.-\left\langle v_{s}^{b}, \phi_{s}^{\lambda}\right\rangle-\lambda \int_{0}^{s}\left\langle v_{s^{\prime}}^{b}, \psi_{b}\right\rangle d s^{\prime}\right)=\exp \left(-\left\langle v_{0}^{b}, \phi_{0}^{\lambda}\right\rangle\right)+\int_{0}^{s} \exp \left(-\left\langle v_{s^{\prime}}^{b}, \phi_{s^{\prime}}^{\lambda}\right\rangle-\lambda \int_{0}^{s^{\prime}}\left\langle v_{r}^{b}, \psi_{b}\right\rangle d r\right) \\
& \times\left(\left\langle v_{s^{\prime}}^{b},-\partial_{s} \phi_{s^{\prime}}^{\lambda}-\frac{1}{2} \Delta \phi_{s^{\prime}}^{\lambda}-\lambda \psi_{b}\right\rangle-\left\langle f\left(v_{s^{\prime}}^{b}\right) \mathbf{1}_{(-\infty,-10 b) \cup(10 b, \infty)}, \phi_{s^{\prime}}^{\lambda}\right\rangle+\frac{1}{2}\left\langle v_{s^{\prime}}^{b}\left(1-v_{s^{\prime}}^{b}\right),\left(\phi_{s^{\prime}}^{\lambda}\right)^{2}\right\rangle\right) d s^{\prime} \\
& +M_{s}^{\phi^{\lambda}, \psi_{b}},
\end{aligned}
$$

where $s \mapsto M_{s}^{\phi^{\lambda}}, \psi_{b}, s \leq t$, is a local martingale. In fact, $M^{\phi^{\lambda}, \psi_{b}}$ is a square integrable martingale: this follows easily from integrability of $\left(\phi^{\lambda}\right)^{2}$. Then we get

$$
\begin{aligned}
\exp ( & \left.-\left\langle v_{s}^{b}, \phi_{s}^{\lambda}\right\rangle-\lambda \int_{0}^{s}\left\langle v_{s^{\prime}}^{b}, \psi_{b}\right\rangle d s^{\prime}\right)=\exp \left(-\left\langle v_{0}^{b}, \phi_{0}^{\lambda}\right\rangle\right)+\int_{0}^{s} \exp \left(-\left\langle v_{s^{\prime}}^{b}, \phi_{s^{\prime}}^{\lambda}\right\rangle-\lambda \int_{0}^{s^{\prime}}\left\langle v_{r}^{b}, \psi_{b}\right\rangle d r\right) \\
& \times\left(\left\langle-f\left(v_{s^{\prime}}^{b}\right) \mathbf{1}_{(-\infty,-10 b) \cup(10 b, \infty)}, \phi_{s^{\prime}}^{\lambda}\right\rangle+\left\langle-\frac{1}{4} v_{s^{\prime}}^{b}+\frac{1}{2} v_{s^{\prime}}^{b}\left(1-v_{s^{\prime}}^{b}\right),\left(\phi_{s^{\prime}}^{\lambda}\right)^{2}\right\rangle\right) d s^{\prime}+M_{s}^{\phi^{\lambda}, \psi_{b}} .
\end{aligned}
$$

Note that (2.25) implies that for $b>R_{0}$ we have a uniform bound

$$
\left|\left\langle v_{0}^{b}, \phi_{0}^{\lambda}\right\rangle\right| \leq c_{0}
$$


with a constant $c_{0}$ that does not depend on $\lambda$. Now we define the stopping times

$$
\tau_{b}=\inf \left\{t \geq 0: \exists x \in[b / 2,3 b] \text { s.t. } v_{t}^{b}(x) \geq \frac{1}{2}\right\}, \quad \rho_{b}=\inf \left\{t \geq 0:\left\langle v_{t}^{b}, \psi_{b}\right\rangle>0\right\} .
$$

Note that we have

$$
\left\langle v_{t \wedge \tau_{b}}^{b}, \phi_{t \wedge \tau_{b}}^{\lambda}\right\rangle+\lambda \int_{0}^{t \wedge \tau_{b}}\left\langle v_{s}^{b}, \psi_{b}\right\rangle d s \rightarrow+\infty \text { as } \lambda \rightarrow+\infty
$$

almost surely on the event $\left\{\rho_{b}<t \wedge \tau_{b}\right)$, thus

$$
\mathbb{P}\left(\rho_{b}<t \wedge \tau_{b}\right) \leq \lim _{\lambda \rightarrow+\infty} \mathbb{E}\left[1-\exp \left(-\left\langle v_{t \wedge \tau_{b}}, \phi_{t \wedge \tau_{b}}^{\lambda}\right\rangle-\lambda \int_{0}^{t \wedge \tau_{b}}\left\langle v_{s}, \psi_{b}\right\rangle d s\right)\right]
$$

On the other hand, taking the expectation in (2.29) with $s=t \wedge \tau_{b}$, we get

$$
\begin{aligned}
\mathbb{E}[1-\exp ( & \left.\left.-\left\langle v_{t \wedge \tau_{b}}^{b}, \phi_{t \wedge \tau_{b}}^{\lambda}\right\rangle-\lambda \int_{0}^{t \wedge \tau_{b}}\left\langle u_{s}, \psi_{b}\right\rangle d s\right)\right] \leq \mathbb{E}\left[1-\exp \left(-\left\langle v_{0}^{b}, \phi_{0}^{\lambda}\right\rangle\right)\right] \\
& +\mathbb{E}\left[\int_{0}^{t}\left(\|f\|_{\infty}\left\langle\mathbf{1}_{(-\infty,-10 b) \cup(10 b, \infty)}, \phi_{s}^{\lambda}\right\rangle+\left\langle\frac{1}{4} v_{s}^{b} \mathbf{1}_{(-\infty, b / 2) \cup(3 b, \infty)},\left(\phi_{s}^{\lambda}\right)^{2}\right\rangle\right) d s\right] .
\end{aligned}
$$

Note that for each $0 \leq s \leq t$ and $x \in \mathbb{R}$ the family $\phi_{s}^{\lambda}(x)$ is increasing in $\lambda$. Moreover, for $s<t$ and $x>b$ we have $\phi_{s}^{\lambda}(x) \rightarrow+\infty$ as $\lambda \rightarrow+\infty$, while for $x<b$, the limit $\phi_{s}^{\infty}(x)$ is finite because of (2.25). Passing to the limit $\lambda \rightarrow+\infty$ in (2.33), using the bound in (2.32) and since $v_{s}^{b}(x) \leq 1$ for all $s \geq 0, x \in \mathbb{R}$, we get

$$
\begin{aligned}
\mathbb{P}\left(\rho_{b}<t \wedge \tau_{b}\right) \leq \mathbb{E}[1 & \left.-\exp \left(-\left\langle v_{0}^{b}, \phi_{0}^{\infty}\right\rangle\right)\right] \\
& +\int_{0}^{t}\left(\|f\|_{\infty}\left\langle\mathbf{1}_{(-\infty,-10 b) \cup(10 b, \infty)}, \phi_{s}^{\infty}\right\rangle+\frac{1}{4}\left\langle\mathbf{1}_{(-\infty, b / 2) \cup(3 b, \infty)},\left(\phi_{s}^{\infty}\right)^{2}\right\rangle\right) d s
\end{aligned}
$$

Recalling (2.27)-(2.28), we have

$$
\begin{aligned}
\mathbb{P}\left(\rho_{b}<t \wedge \tau_{b}\right) \leq & \frac{C}{t} \int_{-\infty}^{0} e^{-(b-x)^{2} /(20 t)} d x \\
& \quad+\frac{\|f\|_{\infty}}{t} \int_{0}^{t}\left(\int_{10 b}^{\infty} e^{-(2 b-x)^{2} /(20 t)} d x+\int_{-\infty}^{-10 b} e^{-(b-x)^{2} /(20 t)} d x\right) d s \\
& \quad+\frac{C}{t^{2}} \int_{0}^{t}\left(\int_{-\infty}^{b / 2} e^{-(b-x)^{2} /(10 t)} d x+\int_{3 b}^{\infty} e^{-(2 b-x)^{2} /(10 t)} d x\right) d s \\
\leq & \frac{C}{b} \exp \left(-\frac{b^{2}}{40 t}\right)+\frac{C\|f\|_{\infty}}{b} \exp \left(-\frac{b^{2}}{t}\right) \leq \frac{C\left(\|f\|_{\infty}+1\right)}{t^{1 / 2}} \exp \left(-\frac{b^{2}}{40 t}\right) .
\end{aligned}
$$

We used the assumption that $R_{0}=0$ in the first term in the right side above. To estimate the integrals in (2.35), we used the standard Gaussian estimate

$$
\int_{y}^{\infty} \exp \left(-x^{2} / 2\right) d x \leq y^{-1} \exp \left(-y^{2} / 2\right)
$$

along with a few changes of variables. 
Now we need to estimate

$$
\begin{aligned}
\mathbb{P}\left(\tau_{b} \leq t\right)=\mathbb{P} & \left(\exists x \in[b / 2,3 b], s \leq t: G_{s} v_{0}^{b}(x)+\int_{0}^{s}\left(\int_{10 b}^{\infty}+\int_{-\infty}^{-10 b}\right) G_{s-r}(x-z) f\left(v_{r}^{b}(z)\right) d z d r\right. \\
& \left.+N_{s}^{b}(x) \geq 1 / 2\right) .
\end{aligned}
$$

It is easy to check that since $b \geq 4 \sqrt{t}\left(t\|f\|_{\infty} \vee 1\right)$

$$
\begin{aligned}
G_{s} v_{0}^{b}(x) & \leq \int_{-\infty}^{0} G_{s}(x-z) d z \leq \int_{-\infty}^{0} G_{t}(x-z) d z \leq \int_{-\infty}^{0} G_{t}(b / 2-z) d z \\
& \leq \int_{-\infty}^{0} G_{1}(2-z) d z \leq 1 / 10, \forall s \leq t, x \in[b / 2,3 b] .
\end{aligned}
$$

Similarly, we have

$$
\begin{aligned}
t \int_{10 b}^{\infty} G_{s-r}(z-x) f\left(v_{r}^{b}(z) d z\right. & \leq t\|f\|_{\infty} \int_{10 b}^{\infty} G_{t}(z-3 b) d z \leq t\|f\|_{\infty} \int_{\frac{7 b}{\sqrt{t}}}^{\infty} G_{1}(z) d z \\
& \leq t\|f\|_{\infty} \int_{28\left(t\|f\|_{\infty} \vee 1\right)}^{\infty} G_{1}(z) d z \leq 0.05, \forall r \leq s \leq t, x \in[b / 2,3 b]
\end{aligned}
$$

and

$$
\begin{aligned}
t \int_{-\infty}^{-10 b} G_{s-r}(z-x) f\left(v_{r}^{b}(z) d z\right. & \leq t\|f\|_{\infty} \int_{-\infty}^{-10 b} G_{t}(z-b / 2) d z \leq t\|f\|_{\infty} \int_{\frac{10 b}{\sqrt{t}}}^{\infty} G_{1}(z) d z, \\
& \leq t\|f\|_{\infty} \int_{40\left(t \mid f \|_{\infty} \vee 1\right)}^{\infty} G_{1}(z) d z \leq 0.05, \forall r \leq s \leq t, x \in[b / 2,3 b]
\end{aligned}
$$

Altogether substituting the last inequalities into (2.36) we get

$$
\begin{aligned}
\mathbb{P}\left(\tau_{b} \leq t\right) & \leq \mathbb{P}\left(\exists x \in[b / 2,3 b], s \leq t: N_{s}^{b}(x) \geq 0.3\right) \\
& \left.\leq C \cdot\left(t \vee t^{22}\right) \int_{\mathbb{R}} \int_{\mathbb{R}} G_{t}(x-z)\left(v_{0}^{b}(z)+t\|f\|_{\infty} \mathbf{1}_{\{z \in(-\infty,-10 b) \cup(10 b, \infty)\}}\right) d z \mathbf{1}_{\{x \in(b / 2,9 b)\}}\right) d x \\
& \leq C\left(t,\|f\|_{\infty}\right) \exp \left(-\frac{b^{2}}{50 t}\right), \forall t>0, x \in[b / 2,3 b],
\end{aligned}
$$

where the second inequality follows by Lemma 2.4 and in the last one we used simple Gaussian bounds. By combining (2.40) with (2.35) we are done.

\section{The proof of Lemma 2.1}

Now we are ready to prove Lemma 2.1. Note that Lemma 2.5] implies a similar result for $u_{t}(x)$.

Lemma 2.6. Let $u_{t}(x)$ be a solution to (1.9) taking values in $\widehat{\mathcal{B}}_{I}$ for all $t \geq 0$ such that the initial condition $u_{0}(x)$ satisfies (1.12) with $R\left(u_{0}\right) \leq 0$. Then, for all $T>0$ there exists $C_{T}>0$ so that for all $b \geq 4 \sqrt{T}\left(T\|f\|_{\infty} \vee 1\right)$ we have

$$
\mathbb{P}\left(\sup _{0 \leq t \leq T} \sup _{x \in[b, 2 b]} u_{t}(x)>0\right) \leq C\left(T,\|f\|_{\infty}\right) \exp \left(-\frac{b^{2}}{50 T}\right) .
$$


Proof. By Girsanov's theorem we have

$$
\begin{aligned}
\mathbb{P}_{u}\left(\sup _{0 \leq t \leq T} \sup _{x \in[b, 2 b]} u_{t}(x)>0\right) & \leq \mathbb{E}_{v^{b}}\left[e^{Z_{T}^{b}} \mathbf{1}_{\left\{\sup _{0 \leq t \leq T} \sup _{x \in[b, 2 b]} v_{t}^{b}(x)>0\right\}}\right] \\
& \leq \mathbb{E}_{v^{b}}\left[e^{2 Z_{T}^{b}}\right] \mathbb{P}_{v^{b}}\left(\sup _{0 \leq t \leq T} \sup _{x \in[b, 2 b]} v_{t}^{b}(x)>0\right),
\end{aligned}
$$

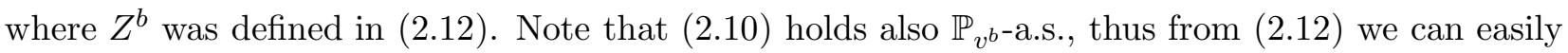
get

$$
\mathbb{E}_{v^{b}}\left[e^{2 Z_{T}^{b}}\right] \leq e^{40 b K_{f}^{2} T},
$$

and combining this with (2.42) and Lemma 2.5] we obtain (2.41).

Now, the conclusions of Lemma 2.1 follow essentially immediately. The bound (2.13) on

$$
\mathbb{P}\left(\sup _{0 \leq t \leq T}\left|R\left(u_{t}\right)-R_{0}\right|>b\right)
$$

in Lemma 2.1 is a simple consequence of Lemma 2.6. The finiteness of $\sup _{t \leq T} R\left(u_{t}\right)$ follows from (2.13) . The corresponding bounds on $L\left(u_{t}\right)$ follow by repeating the arguments used in the proof of Lemmas 2.3 2.6 for $1-u(-x)$ instead of $u(x)$.

\section{Uniqueness of the solution}

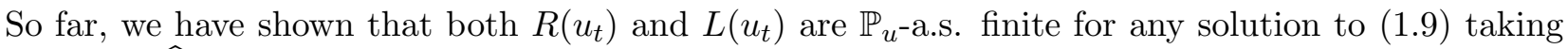
values in $\widehat{\mathcal{B}}_{I}$ for all $t \geq 0$ such that the initial condition $u_{0}(x)$ satisfies (1.12). As a consequence, (2.6) holds for any such solution to (1.9). As we have discussed in Section 2.2, it follows that we may apply Girsanov's theorem to immediately deduce uniqueness in law of the solution to (1.9) that satisfies the above conditions.

\subsection{Existence of the speed}

The last ingredient in the proof of Theorem 1.1 is the existence of the speed.

Lemma 2.7. There exists a deterministic constant $V(\sigma) \in(-\infty,+\infty)$ so that the limit

$$
V(\sigma)=\lim _{t \rightarrow+\infty} \frac{R\left(u_{t}\right)}{t}
$$

exists almost surely.

Proof. The proof goes along the lines of the proof of the corresponding result in [CD05]. First, we show that the limit $V(\sigma)$ in (2.44) exists and $V(\sigma)<\infty$. Let us set $b(m)=R\left(u_{m}\right)$, for $m=0,1,2, \ldots$, and note that by Corollary 2.2 we have

$$
\mathbb{E}\left[(b(1)-b(0))_{+}\right]<\infty .
$$

Then, as in the proof of Lemma 5.1 in [CD05] we can use the subadditive ergodic theorem to deduce that there exists a constant $c(\sigma) \in[-\infty, \infty)$, such that

$$
\lim _{m \rightarrow+\infty} \frac{b(m)}{m}=c(\sigma) .
$$


Using Lemma 2.1, we get (see Lemma 5.3 in CD05] for the same argument) that for all $m=1,2, \ldots$

$$
\mathbb{P}\left(\sup _{0 \leq s \leq 1}\{b(s+m)-b(m), b(m+1)-b(s+m)\}>\sqrt{m}\right) \leq C(\sigma) \exp (-m / 50) .
$$

Then by the Borel-Cantelli lemma we get that in fact,

$$
\lim _{t \rightarrow+\infty} \frac{b(t)}{t}=c(\sigma)
$$

and thus $V(\sigma)=c(\sigma)<\infty$.

To show that $V(\sigma)>-\infty$, one needs to consider equation for $1-u_{t}(-x)$ and repeat the above argument.

\section{The interface in the voter model}

Girsanov's theorem connecting solutions to the rescaled equation (1.14) and to the voter model (1.15) not only allows us to deduce uniqueness in the law for the solutions to the former problem but also obtain the asymptotics on their front speed in Theorem 1.2. As a preliminary step, in this section, we make some observations about the latter. To begin, we rephrase Lemma 4.2(a) of [Tri95, putting it into a form more directly useful for our purposes. Let $w_{t}(x)$ be the solution to (1.15) with an initial condition $w_{0}(x)$ satisfying (1.12). Recall that we denote by $\mathbb{P}_{w}$ the measure induced on the canonical path space $C([0,+\infty) ; C(\mathbb{R}))$ by $w$, and by $\mathbb{E}_{w}$ we denote the corresponding expectation. Recall that two random processes $X_{t}$ and $Y_{t}$ are said to be coupled if they can be defined on the same probability space. We assume throughout the rest of the paper that $f$ satisfies assumption (1.16).

Lemma 3.1. Given $\varepsilon>0$, there exists $T_{\varepsilon}>0$ such that for all $T \geq T_{\varepsilon}$ there is a coupling of processes $\left(w_{t}, B_{t}: t \geq 0\right)$ where $B$ a standard Brownian motion started at 0 , such that

$$
\mathbb{P}_{w}\left(\sup _{0 \leq t \leq T}\left|R\left(w_{t}\right)-B_{t}\right| \vee\left|L\left(w_{t}\right)-B_{t}\right| \geq T^{1 / 2} \varepsilon\right) \leq \varepsilon
$$

The following lemma shows that another good measure of the location of the interface is

$$
M_{t}:=\int_{0}^{t} \int_{\mathbb{R}} \sqrt{w_{s}(x)\left(1-w_{s}(x)\right)} W(d x, d s) .
$$

Lemma 3.2. Let $B$ be the Brownian motion from Lemma 3.1. Given $\varepsilon>0$, there exists $T_{\varepsilon}>0$ such that for all $T \geq T_{\varepsilon}$ we have

$$
\mathbb{P}_{w}\left(\sup _{0 \leq t \leq T}\left|M_{t}-B_{t}\right| \geq 4 T^{1 / 2} \varepsilon\right) \leq \varepsilon
$$

Proof. By Lemma 3.1 .

$$
\Xi\left(w_{t}\right):=\int_{-\infty}^{0}\left[w_{t}(x)-1\right] d x+\int_{0}^{\infty} w_{t}(x) d x .
$$

is an almost surely finite functional of $w_{t}$. As $w_{t}(x)=1$ for $x<L\left(w_{t}\right)$ and $w_{t}(x)=0$ for $x>R\left(w_{t}\right)$, we have

$$
\Xi\left(w_{t}\right)=\int_{L\left(w_{t}\right) \wedge 0}^{0}\left[w_{t}(x)-1\right] d x+\int_{0}^{R\left(w_{t}\right) \vee 0} w_{t}(x) d x,
$$


thus

$$
L\left(w_{t}\right)=\int_{L\left(w_{t}\right) \wedge 0}^{0}[-1] d x+\int_{0}^{L\left(w_{t}\right) \vee 0} d x \leq \int_{L\left(w_{t}\right) \wedge 0}^{0}\left[w_{t}(x)-1\right] d x+\int_{0}^{R\left(w_{t}\right) \vee 0} w_{t}(x) d x=\Xi\left(w_{t}\right),
$$

and likewise

$$
R\left(w_{t}\right)=\int_{R\left(w_{t}\right) \wedge 0}^{0}[-1] d x+\int_{0}^{R\left(w_{t}\right) \vee 0} d x \geq \int_{L\left(w_{t}\right) \wedge 0}^{0}\left[w_{t}(x)-1\right] d x+\int_{0}^{R\left(w_{t}\right) \vee 0} w_{t}(x) d x=\Xi\left(w_{t}\right) .
$$

We conclude that

$$
\left.L\left(w_{t}\right)\right) \leq \Xi\left(w_{t}\right) \leq R\left(w_{t}\right) .
$$

Next, let $\theta(x)$ be a smooth monotonically decreasing function such that $\theta(x)=1$ for $x<-2$ and $\theta(x)=0$ for $x>-1$, and set $\theta_{n}(x)=\theta(n x)$. Then for

$$
\zeta_{n}(x):=w_{t}(x)-\theta_{n}(x)
$$

we have

$$
\Xi\left(w_{t}\right)=\lim _{n \rightarrow \infty} \Xi_{n}(t), \quad \Xi_{n}(t)=\int_{-\infty}^{\infty} \zeta_{n}(x) d x .
$$

The function $\zeta_{n}(t, x)$ satisfies

$$
\partial_{t} \zeta_{n}=\frac{1}{2} \partial_{x}^{2} \zeta_{n}+\frac{1}{2} \partial_{x}^{2} \theta_{n}+\sqrt{w(1-w)} \dot{W}(t, x) .
$$

Integrating in $t$ and $x$ gives

$$
\Xi_{n}\left(w_{t}\right)=\Xi_{n}\left(w_{0}\right)+\int_{0}^{t} \int_{\mathbb{R}} \sqrt{w_{s}(y)\left(1-w_{s}(y)\right)} W(d y d s) .
$$

Passing to the limit $n \rightarrow+\infty$, we arrive at

$$
\Xi\left(w_{t}\right)=\Xi\left(w_{0}\right)+\int_{0}^{t} \int_{\mathbb{R}} \sqrt{w_{s}(y)\left(1-w_{s}(y)\right)} W(d y d s)=\Xi\left(w_{0}\right)+M_{t} .
$$

As $\Xi\left(w_{0}\right)<+\infty$ and is not random, the conclusion of the present lemma follows from Lemma 3.1 by taking $T_{\varepsilon}$ sufficiently large.

For any metric space $\mathbf{E}$, we denote by $\mathbb{D}_{\mathbf{E}}$ the space of càdlàg functions $[0, \infty) \rightarrow \mathbf{E}$ equipped with the Skorohod topology. Define the rescaled functionals

$$
L_{t}^{a}=\frac{1}{a} L\left(w_{a^{2} t}\right), R_{t}^{a}=\frac{1}{a} R\left(w_{a^{2} t}\right), \quad M_{t}^{a}=\frac{1}{a} M_{a^{2} t} .
$$

As a consequence of Lemmas 3.1 and 3.2, we conclude that

$$
\left(L^{a}, R^{a}, M^{a}\right) \Rightarrow(B, B, B) \text { in } \mathbb{D}_{\mathbb{R}^{3}}, \text { as } a \rightarrow \infty,
$$

where $B$ is a standard Brownian motion starting at 0 and $\Rightarrow$ denotes convergence in law.

As in the application of the Girsanov theorem in the proof of Theorem 1.1, we will make use of the functionals

$$
\begin{aligned}
A_{t}^{f} & :=\int_{0}^{t} \int_{\mathbb{R}} \frac{f\left(w_{s}(x)\right)^{2}}{w_{s}(x)\left(1-w_{s}(x)\right)} d x d s, \\
M_{t}^{f} & :=\int_{0}^{t} \int_{\mathbb{R}} \frac{f\left(w_{s}(x)\right)}{\sqrt{w_{s}(x)\left(1-w_{s}(x)\right)}} W(d x, d s),
\end{aligned}
$$


and their rescaled versions

$$
M_{t}^{f, a}=\frac{1}{a} M_{a^{2} t}, \quad A_{t}^{f, a}=\frac{1}{a^{2}} A_{a^{2} t}^{f}, \quad a>0 .
$$

The difference in the scaling of these two functionals comes from the fact that $M_{t}$ is, roughly, a Brownian motion on large time scales, and $A_{t}$ is deterministic to the leading order on large time scales. Note that both $A_{t}$ and $M_{t}$ are almost surely finite if $f$ satisfies assumption (1.11), since the interface of $w_{t}$ has a finite length almost surely. However, we will need the stronger assumption (1.16) in Lemma 3.5 below.

Let us now recall Theorem 1 of [MT97].

Theorem 3.3 ([MT97]). There exists a unique stationary measure $\mu$ on $\mathcal{C}_{I}$ for (1.15). Furthermore, for each $u_{0} \in \mathcal{C}_{I}$, the law of $w_{t}\left(x+L_{t}\right)$ converges in total variation to $\mu$ as $t \rightarrow \infty$. In addition, the moment of the width of the interface $\mathbb{E}_{w, s t}[R(f)-L(f)]^{p} \mu(d f)$ is finite if $0 \leq p<1$, and infinite for $p \geq 1$.

The following estimate is a consequence of the second part of Theorem 3.3 .

Lemma 3.4. For any $\eta \in(0,1]$, we have

$$
\mathbb{E}_{w, s t}\left[\int_{\mathbb{R}}(w(x)(1-w(x)))^{\eta} d x\right]<\infty .
$$

Note that this result fails at $\eta=0$ : according to Theorem 3.3, the length of the interface has an infinite expectation under the stationary distribution of $w$.

Proof. For $\eta=1$ the result is known (see Lemma 2.1(a) in [Tri95]), so we assume that $\eta \in(0,1)$. Let $\ell$ be the length of the interface of $w$ under the stationary distribution. By applying Hölder's and Young's inequalities we get

$$
\begin{aligned}
\mathbb{E}_{w, s t}\left[\int_{\mathbb{R}}(w(x)(1-w(x)))^{\eta} d x\right] & \leq \mathbb{E}_{w, s t}\left[\left(\int_{\mathbb{R}}(w(x)(1-w(x))) d x\right)^{\eta} \ell^{1-\eta}\right] \\
& \leq C_{\alpha} \mathbb{E}_{w, s t}\left[\left(\int_{\mathbb{R}}(w(x)(1-w(x))) d x\right)^{\alpha \eta}\right]+C_{\alpha} \mathbb{E}_{w, s t}\left[\ell^{\frac{\alpha(1-\eta)}{\alpha-1}}\right],
\end{aligned}
$$

for any $\alpha>1$. We take $\alpha=2 / \eta$ and get

$$
\mathbb{E}_{w, s t}\left[\int_{\mathbb{R}}(w(x)(1-w(x)))^{\eta} d x\right] \leq C_{\alpha} \mathbb{E}_{w, s t}\left[\left(\int_{\mathbb{R}}(w(x)(1-w(x))) d x\right)^{2}\right]+C_{\alpha} \mathbb{E}_{w, s t}\left[\ell^{\gamma}\right],
$$

with $\gamma=(1-\eta) /(1-\eta / 2)$. Since $\gamma<1$, by Theorem 3.3 we get $\mathbb{E}_{w, s t}\left[\ell^{\gamma}\right]<\infty$. In addition, Lemma 2.1(d) in [Tri95] implies that

$$
\mathbb{E}_{w, s t}\left[\left(\int_{\mathbb{R}}(w(x)(1-w(x))) d x\right)^{2}\right]<\infty,
$$

and we are done.

Lemma 3.5. Let $f$ satisfy assumption (1.16), then we have convergence in law

$$
\left.\left(M^{f, a}, A^{f, a}\right) \Rightarrow\left\{B_{t}^{f}, D t\right), t \geq 0\right\},
$$

in $\mathbb{D}_{\mathbb{R}^{2}}$, as $a \rightarrow \infty$. Here $\left\{B_{t}^{f}, t \geq 0\right\}$ is a Brownian motion with variance $D$

$$
D=\mathbb{E}_{w, s t}\left[\int_{\mathbb{R}} \frac{f(w(x))^{2}}{w(x)(1-w(x))} d x\right]<\infty .
$$


Note that $D<+\infty$ because of Lemma 3.4 and assumption (1.16) on $f$.

Proof. Since $w$ has a unique stationary distribution on the space $\mathcal{C}_{I}$ of continuous functions $h$ such that $-\infty<L(h)<R(h)<+\infty$, by the ergodic theorem we have

$$
\lim _{a \rightarrow \infty} a^{-2} A_{a^{2} t}^{f}=t \mathbb{E}_{w, s t}\left[\int_{\mathbb{R}} \frac{f(w(x))^{2}}{w(1-w(x))} d x\right]=D t,
$$

uniformly on compact sets in $t$. Recall that $\mathbb{E}_{w, s t}$ denotes the expectation with respect to the stationary measure of $w$ on $\mathcal{C}_{I}$. Since

$$
M_{t}^{f, a}=\tilde{B}_{A_{t}^{f, a}}, t \geq 0
$$

for some standard Brownian motion $\tilde{B}$, it follows from (3.12) that

$$
M^{f, a} \Rightarrow\left\{B_{t}^{f}, t \geq 0\right\}:=\left\{\tilde{B}_{D t}, t \geq 0\right\},
$$

where $\tilde{B}_{D t}$ is a Brownian motion with variance $D$.

Define

$$
A_{t}:=\int_{0}^{t} \int_{\mathbb{R}} w_{s}(x)\left(1-w_{s}(x)\right) d x d s
$$

and its rescaled version

$$
A_{t}^{a}=\frac{1}{a^{2}} A_{a^{2} t}, \quad a>0 .
$$

Corollary 3.6. We have convergence in law

$$
\left(L^{a}, R^{a}, M^{a}, A^{a}, M^{f, a}, A^{f, a}\right) \Rightarrow\left\{\left(B_{t}, B_{t}, B_{t}, t, B_{t}^{f}, D t\right), t \geq 0\right\}
$$

in $\mathbb{D}_{\mathbb{R}^{5}}$, as a $\rightarrow \infty$. Here, $B_{t}$ is a standard Brownian motion, $B_{t}^{f}$ is a Brownian motion with variance $D$ and their correlation is given by

$$
\left\langle B ., B^{f}\right\rangle_{t}=c_{f} t, t \geq 0,
$$

with $c_{f}$ as in (1.18).

Proof. It only remains to check the correlation:

$$
\left\langle M_{\cdot}^{f, a}, M_{\cdot}^{a}\right\rangle_{t}=a^{-2} \int_{0}^{a^{2} t} \int_{\mathbb{R}} f\left(w_{s}(x)\right) d x d s \Rightarrow t \mathbb{E}_{w, s t}\left[\int_{\mathbb{R}} f(w(x)) d x\right]=c_{f} t, t \geq 0,
$$

as $a \rightarrow \infty$, exactly as in (3.12).

\section{The proof of Theorem 1.2: the upper bound on the speed}

We assume till the end of the paper, without loss of generality, that $c_{f}>0$. In this section, we prove the upper bound on the front speed in Theorem 1.2 ,

Proposition 4.1. Suppose that $u_{0}$ satisfies (1.12) and $f$ satisfies (1.16). Then with probability 1, we have

$$
\limsup _{\sigma \rightarrow \infty} \sigma^{2} V(\sigma) \leq c_{f} .
$$




\subsection{Rescaling}

First, we show via a rescaling how to pass from (1.9) to (1.14). Consider the rescaled function

$$
v_{t}(x)=u_{\sigma^{-4} t}\left(\sigma^{-2} x\right) \text {. }
$$

To get an equation for $v_{t}(x)$, we use the mild form (2.1) and the relations

$$
\begin{aligned}
G_{a^{2} t}(b x) & =b^{-1} G_{\left(a^{2} t / b^{2}\right)}(x) \\
W^{a, b}(d y d s) & :=a^{-1} b^{-1 / 2} W\left(b d y, a^{2} d s\right) \stackrel{\mathcal{D}}{=} W(d y d s) \\
a b^{1 / 2} W^{a, b}(d y d s) & =W\left(b d y, a^{2} d s\right),
\end{aligned}
$$

that hold for any $a, b>0$. Here, $\stackrel{\mathcal{D}}{=}$ means equality in distribution. From (2.1), for any $a, b>0$, we get

$$
\begin{aligned}
u_{\left(a^{2} t\right)}(b x)= & \int_{\mathbb{R}} G_{a^{2} t}(b x-y) u_{0}(y) d y+\int_{0}^{a^{2} t} \int_{\mathbb{R}} G_{a^{2} t-s}(b x-y) f\left(u_{s}(y)\right) d y d s \\
& +\sigma \int_{0}^{a^{2} t} \int_{\mathbb{R}} G_{a^{2} t-s}(b x-y) \sqrt{u_{s}(y)\left(1-u_{s}(y)\right)} W(d y d s)=: I+I I+I I I .
\end{aligned}
$$

We make the change of variables $s=a^{2} s^{\prime}, y=b y^{\prime}$ and use (4.1). For the term $I$ we have

$$
I=b \int_{\mathbb{R}} G_{a^{2} t}\left(b x-b y^{\prime}\right) u_{0}\left(b y^{\prime}\right) d y^{\prime}=\int_{\mathbb{R}} G_{a^{2} t / b^{2}}\left(x-y^{\prime}\right) u_{0}\left(b y^{\prime}\right) d y^{\prime} .
$$

The second term can be rewritten as

$$
\begin{aligned}
I I & =\int_{0}^{a^{2} t} \int_{\mathbb{R}} G_{a^{2} t-s}(b x-y) f\left(u_{s}(y)\right) d y d s=\int_{0}^{t} \int_{\mathbb{R}} G_{a^{2} t-a^{2} s^{\prime}}\left(b x-b y^{\prime}\right) f\left(u_{a^{2} s^{\prime}}\left(b y^{\prime}\right)\right) b a^{2} d y^{\prime} d s^{\prime} \\
& =a^{2} \int_{0}^{t} \int_{\mathbb{R}} G_{a^{2}\left(t-s^{\prime}\right) / b^{2}}\left(x-y^{\prime}\right) f\left(u_{a^{2} s^{\prime}}\left(b y^{\prime}\right)\right) d y^{\prime} d s^{\prime}
\end{aligned}
$$

and changing variables, the last term is

$$
\begin{aligned}
I I I & =\sigma \int_{0}^{a^{2} t} \int_{\mathbb{R}} G_{a^{2} t-s}(b x-y) \sqrt{u_{s}(y)\left(1-u_{s}(y)\right)} W(d y d s) \\
& =\sigma \int_{0}^{t} \int_{\mathbb{R}} G_{a^{2} t-a^{2} s^{\prime}}\left(b x-b y^{\prime}\right) \sqrt{u_{a^{2} s^{\prime}}\left(b y^{\prime}\right)\left(1-u_{a^{2} s^{\prime}}\left(b y^{\prime}\right)\right)} W\left(b d y^{\prime}, a^{2} d s^{\prime}\right) \\
& =\sigma \int_{0}^{t} \int_{\mathbb{R}} b^{-1} G_{a^{2}\left(t-s^{\prime}\right) / b^{2}}\left(x-y^{\prime}\right) \sqrt{u_{a^{2} s^{\prime}}\left(b y^{\prime}\right)\left(1-u_{a^{2} s^{\prime}}\left(b y^{\prime}\right)\right)} a b^{1 / 2} W^{a, b}\left(d y^{\prime} d s^{\prime}\right) . \\
& =a b^{-1 / 2} \sigma \int_{0}^{t} \int_{\mathbb{R}} G_{a^{2}\left(t-s^{\prime}\right) / b^{2}}\left(x-y^{\prime}\right) \sqrt{u_{a^{2} s^{\prime}}\left(b y^{\prime}\right)\left(1-u_{a^{2} s^{\prime}}\left(b y^{\prime}\right)\right)} W^{a, b}\left(d y^{\prime} d s^{\prime}\right) .
\end{aligned}
$$

We take

$$
a=\sigma^{-2}, \quad b=\sigma^{-2},
$$

so that $a b^{-1 / 2} \sigma=1$ and $a^{2} / b^{2}=1$. Defining $v_{t}(x):=u_{\left(a^{2} t\right)}(b x)$ and putting together the above terms, we see that $v_{t}(x)$ satisfies

$$
\begin{aligned}
v_{t}(x)= & \int_{\mathbb{R}} G_{t}(x-y) u_{0}(y) d y+\sigma^{-4} \int_{0}^{t} \int_{\mathbb{R}} G_{t-s}(x-y) f\left(v_{s}(y)\right) d y d s \\
& +\int_{0}^{t} \int_{\mathbb{R}} G_{t-s}(x-y) \sqrt{v_{s}(y)\left(1-v_{s}(y)\right)} W^{a, b}(d y d s) .
\end{aligned}
$$


Since the solution $v$ to (4.5) is unique in law, and since $W$ and $W^{a, b}$ are equal in law, we see that $v$ is the unique weak solution to (1.14) with the initial condition $v_{0}(x)=u_{0}\left(\sigma^{-2} x\right)$. Thus in general our scaling changes the initial data. However, if $u_{0}(x)=\mathbf{1}(x \leq 0)$, then clearly $v_{0}(x)=u_{0}(x)$.

Now it is clear that the conclusion of Proposition 4.1 would follow if we show that

$$
\limsup _{\sigma \rightarrow \infty} \sigma^{4} V^{(v)}(\sigma) \leq c_{f}
$$

where

$$
V^{(v)}(\sigma)=\lim _{t \rightarrow+\infty} \frac{R\left(v_{t}\right)}{t} .
$$

Let us also note that the rescaled Girsanov functional (2.4) takes the form

$$
\begin{aligned}
Z_{t} & =\sigma^{-4} \int_{0}^{t} \int_{\mathbb{R}} \frac{f\left(w_{s}(x)\right)}{\sqrt{w_{s}(x)\left(1-w_{s}(x)\right)}} W(d x, d s)-\frac{1}{2} \sigma^{-8} \int_{0}^{t} \int_{\mathbb{R}} \frac{f\left(w_{s}(x)\right)^{2}}{w_{s}\left((x) 1-w_{s}(x)\right)} d x d s \\
& =\sigma^{-4} M_{t}^{f}-\frac{1}{2} \sigma^{-8} A_{t}^{f} .
\end{aligned}
$$

\subsection{Time steps for the upper bound}

Note that for the upper bound on $V(\sigma)$ and $V^{(v)}(\sigma)$, we may assume without loss of generality that the initial condition $u_{0}(x)=v_{0}(x)=\mathbf{1}(x \leq 0)$, by the comparison principle and translation invariance in law. We will define a sequence of stopping times $0=\tau_{0} \leq \tau_{1} \leq \cdots$, and a sequence $v_{t}^{(m)}(x)$ of solutions to (1.14) for $t \geq \tau_{m}$, with the initial conditions $v_{\tau_{m}}^{(m)}(x) \geq v_{\tau_{m}}(x)$ at $t=\tau_{m}$. The comparison principle will imply that $v_{t}^{(m)}(x) \geq v_{t}(x)$ for $t \geq \tau_{m}$. Moreover, we will choose $v_{t}^{(m)}$ so that for each $m=0,1,2, \ldots$ the following conditions hold almost surely:

$$
\begin{aligned}
v_{t}(x) & \leq v_{t}^{(m)}(x), \quad \text { for } t \geq \tau_{m}, x \in \mathbb{R} \\
R\left(v_{\tau_{m}}^{(m)}\right) & =m \lambda_{1} \sigma^{4}, \\
v_{\tau_{m}}^{(m-1)}(x) & \leq v_{\tau_{m}}^{(m)}(x), \quad \text { for } x \in \mathbb{R},
\end{aligned}
$$

with the constant $\lambda_{1}$ to be specified later. It follows from (4.8), that for all $m=0,1,2, \ldots$ and for all $t \geq \tau_{m}$, we have

$$
R\left(v_{t}\right) \leq R\left(v_{t}^{(m)}\right)
$$

almost surely. Thus, to bound $R\left(v_{t}\right)$ from above, it suffices to bound $R\left(v_{t}^{(m)}\right)$.

Let us inductively construct $\tau_{k}$ and $v_{t}^{(k)}(x)$ for $k=0,1, \ldots$ For convenience in (4.10), we define $v_{t}^{(-1)}(x)=0$. Fix $T_{0}>0$ and $N \in \mathbb{N}$, to be specified later, and start with $\tau_{0}=0$ and $v_{t}^{(0)}(x)=v_{t}(x)$, so that (4.8), (4.9), and (4.10) hold for $m=0$ automatically. Suppose that we have defined $\tau_{m}$ and $v_{t}^{(m)}$ for $t \geq \tau_{m}$ and $0 \leq m \leq k$, and assume that (4.8), (4.9) and (4.10) hold for $0 \leq m \leq k$. Given $v_{t}^{(k)}(x)$, defined for $t \geq \tau_{k}$ and $x \in \mathbb{R}$, we set

$$
M_{t}^{f, v, k}:=\int_{\tau_{k}}^{t} \int_{\mathbb{R}} \frac{f\left(v_{s}^{(k)}(x)\right)}{\sqrt{v_{s}^{(k)}(x)\left(1-v_{s}^{(k)}(x)\right)}} W(d x, d s),
$$

and

$$
\tau_{k+1}=\inf \left\{t \in\left[\tau_{k}, \tau_{k}+T_{0} \sigma^{8}\right]: R\left(v_{t}^{(k)}\right)=(k+1) \lambda_{1} \sigma^{4} \text { or } \quad \frac{1}{\sigma^{4}} M_{t}^{f, v, k} \geq N\right\}
$$


with $\tau_{k+1}=\tau_{k}+T_{0} \sigma^{8}$ if the above set is empty. Then, we define $v_{t}^{(k+1)}(x)$ for $t \geq \tau_{k+1}$, and $x \in \mathbb{R}$ as the solution to (1.14) with the initial condition

$$
v_{\tau_{k+1}}^{(k+1)}(x)=\mathbf{1}\left(x \leq(k+1) \lambda_{1} \sigma^{4}\right) .
$$

Note that for $m=k+1$, (4.8) and (4.10) hold by the comparison principle. (4.9) holds by construction.

For convenience, we write

$$
\Delta \tau_{m}=\tau_{m+1}-\tau_{m}
$$

and note that $\left\{\Delta \tau_{m}\right\}$ are i.i.d. random variables for $m \geq 0$.

\subsection{A good event and its consequences (for the upper bound)}

To get an upper bound on $V_{f}^{(v)}(\sigma)$, it suffices to get an appropriate lower bound on $\tau_{m}$ as $m \rightarrow \infty$. Let us define the event

$$
G_{m}=\left\{\Delta \tau_{m}=T_{0} \sigma^{8}\right\}, \quad m \geq 0 .
$$

Proposition 4.1 is a consequence of the following lemma.

Lemma 4.2. Let $\varepsilon \in\left(0, \min \left(10^{-1}, c_{f}^{-2}\right)\right)$ be arbitrary and set $\delta_{\varepsilon}=\varepsilon / 10$. There exist $\bar{T}_{\varepsilon}, N_{\varepsilon}$, and $\sigma_{\varepsilon}$ such that for $T_{0}=\bar{T}_{\varepsilon}, N=N_{\varepsilon}$ and any $\sigma \geq \sigma_{0}^{\varepsilon}, m \geq 0$, and

$$
\lambda_{1}=\left(c_{f}+\delta_{\varepsilon}\right) \bar{T}_{\varepsilon},
$$

we have

$$
\lambda_{2}:=\mathbb{P}_{v}\left(G_{m}\right) \geq 1-\delta_{\varepsilon} .
$$

Note that $\lambda_{2}$ does not depend on $m$ since $\Delta \tau_{m}$ are i.i.d for $m \geq 1$. We will prove Lemma 4.2 in the next section. Now we are ready to give

Proof of Proposition 4.1. Given $\varepsilon \in(0,1 / 10)$, let $\bar{T}_{\varepsilon}, N_{\varepsilon}$ and $\sigma_{\varepsilon}$ be as in Lemma 4.2, and take an arbitrary $\sigma \geq \sigma_{\varepsilon}$. Then by Lemma 4.2 , we have

$$
\lambda_{2} \geq 1-\delta_{\varepsilon}
$$

and by (4.16) and the definition of $G^{(m)}$ with $T_{0}=\bar{T}_{\varepsilon}$, we get

$$
\mathbb{E}_{v}\left[\Delta \tau_{m}\right] \geq \bar{T}_{\varepsilon} \lambda_{2} \sigma^{8}
$$

The strong law of large numbers implies that we have, $\mathbb{P}_{v}$ almost surely,

$$
\lim _{m \rightarrow \infty} \frac{\tau_{m}}{m} \geq \bar{T}_{\varepsilon} \lambda_{2} \sigma^{8}
$$

Since $R\left(v_{\tau_{m}}^{(m)}\right)=m \lambda_{1} \sigma^{4}$, we have that, also $\mathbb{P}_{v}$ almost surely,

$$
\limsup _{m \rightarrow \infty} \frac{R\left(v_{\tau_{m}}^{(m)}\right)}{\tau_{m}}=\limsup _{m \rightarrow \infty} \frac{m \lambda_{1} \sigma^{4}}{\tau_{m}} \leq \frac{\lambda_{1} \sigma^{4}}{\bar{T}_{\varepsilon} \lambda_{2} \sigma^{8}}=\frac{\lambda_{1}}{\bar{T}_{\varepsilon} \lambda_{2}} \sigma^{-4} .
$$

Furthermore, since by definition, for $\tau_{m} \leq t \leq \tau_{m+1}$ we have

$$
R\left(v_{t}^{(m)}\right) \leq(m+1) \lambda_{1} \sigma^{4}
$$


Hence, we get that, $\mathbb{P}_{v}$ almost surely, we have, using (4.17) and (4.18),

$$
\begin{aligned}
V^{(v)}(\sigma) & \leq \limsup _{m \rightarrow \infty} \sup _{\tau_{m} \leq t \leq \tau_{m+1}} \leq \frac{R\left(v_{t}^{(m)}\right)}{t} \leq \limsup _{m \rightarrow \infty} \frac{\lambda_{1}(m+1) \sigma^{4}}{\tau_{m}} \\
& \leq \frac{\lambda_{1} \sigma^{4}}{\lambda_{2} \bar{T}_{\varepsilon} \sigma^{8}} \leq \frac{\left(c_{f}+\delta_{\varepsilon}\right)}{\left(1-\delta_{\varepsilon}\right)} \sigma^{-4} \leq\left(c_{f}+\sqrt{\varepsilon}\right) \sigma^{-4} .
\end{aligned}
$$

Note that (4.20) holds for any $\sigma \geq \sigma_{0}^{\varepsilon}$, and, since $\varepsilon$ is arbitrary small, we are done. This finishes the proof of Proposition 4.1.

\subsection{Proof of Lemma 4.2}

As $G_{m}$ are i.i.d., it suffices to set $m=0$. We fix $\varepsilon \in(0,1 / 10)$, let $\delta_{\varepsilon}=\varepsilon / 10$, take $\bar{T}_{\varepsilon}$ sufficiently large, so that

$$
2 \exp \left(-\frac{\delta_{\varepsilon}^{2} \bar{T}_{\varepsilon}}{2}\right) \leq \frac{\varepsilon}{100}
$$

set $\lambda_{1}=\left(c_{f}+\delta_{\varepsilon}\right) \bar{T}_{\varepsilon}$, and let $N_{\varepsilon}>\left(2+\delta_{\varepsilon}\right) \bar{T}_{\varepsilon} D$ be sufficiently large (its value will be determined later in the proof). We define the stopping time

$$
\xi^{\varepsilon}=\inf \left\{t \geq 0: M_{t}^{f, \sigma^{4}} \geq N_{\varepsilon}\right\}
$$

Then by Girsanov's theorem, we have, with $Z_{t}$ as in (4.7):

$$
\begin{aligned}
& \mathbb{P}_{v}\left(G_{0}^{c}\right)=\mathbb{E}_{w}\left[\exp \left(Z_{\sigma^{8}\left(\bar{T}_{\varepsilon} \wedge \xi^{\varepsilon}\right)}\right) \mathbf{1}_{G_{0}^{c}}\right]=\mathbb{E}_{w}\left[\exp \left(\sigma^{-4}\left(M_{\sigma^{8}\left(\bar{T}_{\varepsilon} \wedge \xi^{\varepsilon}\right)}^{f}-\frac{1}{2} \sigma^{-4} A_{\sigma^{8}\left(\bar{T}_{\varepsilon} \wedge \xi^{\varepsilon}\right)}^{f}\right)\right)\right. \\
&\left.\quad \times \mathbf{1}\left(R\left(w_{t}\right) \geq \lambda_{1} \sigma^{4} \text { for some } t \leq \sigma^{8} \bar{T}_{\varepsilon} \text { or } \sigma^{-4} M_{t}^{f} \geq N_{\varepsilon} \text { for some } t \leq \sigma^{8} \bar{T}_{\varepsilon}\right)\right] \\
&=\mathbb{E}_{w}\left[\exp \left(M_{\bar{T}_{\varepsilon} \wedge \xi^{\varepsilon}}^{f, \sigma^{4}}-\frac{1}{2} A_{\bar{T}_{\varepsilon} \wedge \xi^{\varepsilon}}^{f, \sigma^{4}}\right) \times \mathbf{1}\left(R_{t}^{\sigma^{4}} \geq\left(c_{f}+\delta_{\varepsilon}\right) \bar{T}_{\varepsilon} \text { for some } t \leq \bar{T}_{\varepsilon} \text { or } \xi^{\varepsilon} \leq \bar{T}_{\varepsilon}\right)\right] \\
& \leq \mathbb{E}_{w}\left[\exp \left(M_{\bar{T}_{\varepsilon} \wedge \xi^{\varepsilon}}^{f, \sigma^{4}}-\frac{1}{2} A_{\bar{T}_{\varepsilon} \wedge \xi^{\varepsilon}}^{f, \sigma^{4}}\right) \mathbf{1}\left(\xi^{\varepsilon} \leq \bar{T}_{\varepsilon}\right)\right] \\
&+\mathbb{E}_{w}\left[\exp \left(M_{\bar{T}_{\varepsilon} \wedge \xi^{N, \sigma}}^{\sigma^{4}}-\frac{1}{2} A_{\bar{T}_{\varepsilon} \wedge \xi^{N, \sigma}}^{f, \sigma^{4}}\right) \times \mathbf{1}\left(R_{t}^{\sigma^{4}} \geq\left(c_{f}+\delta_{\varepsilon}\right) \bar{T}_{\varepsilon} \text { for some } t \leq \bar{T}_{\varepsilon}\right) \mathbf{1}\left(\xi^{\varepsilon}>T_{\varepsilon}\right)\right] \\
&=: I_{1}^{\varepsilon}+I_{2}^{\varepsilon} .
\end{aligned}
$$

We first bound $I_{1}^{\varepsilon}$ :

$$
I_{1}^{\varepsilon}=\mathbb{E}_{w}\left[\exp \left(M_{\xi^{\varepsilon} \wedge \bar{T}_{\varepsilon}}^{f, \bar{\sigma}^{4}}-\frac{1}{2} A_{\xi^{\varepsilon} \wedge \bar{T}_{\varepsilon}}^{f, \sigma^{4}}\right) \mathbf{1}\left(\xi^{\varepsilon} \leq \bar{T}_{\varepsilon}\right)\right] \leq e^{N_{\varepsilon}} \mathbb{P}_{w}\left(\sup _{0 \leq t \leq \bar{T}_{\varepsilon}} M_{t}^{f, \sigma^{4}} \geq N_{\varepsilon}\right) .
$$

Let $\mathbb{P}^{B}$ and $\mathbb{P}^{B^{f}}$ be the measures induced on the canonical path space by the standard Brownian motion $B$ and by the Brownian motion with variance $D$, respectively, and $\mathbb{E}^{B}$ and $\mathbb{E}^{B^{f}}$ be the corresponding expectations. Then by Lemma 3.5 we have

$$
\limsup _{\sigma \rightarrow \infty} I_{1}^{\varepsilon} \leq e^{N_{\varepsilon}} \mathbb{P}^{B^{f}}\left(\sup _{0 \leq t \leq \bar{T}_{\varepsilon}} B_{t}^{f} \geq N_{\varepsilon}\right) \leq e^{N_{\varepsilon}} 2 \mathbb{P}^{B}\left(\sqrt{D} B_{\bar{T}_{\varepsilon}} \geq N_{\varepsilon}\right) \leq 2 e^{N_{\varepsilon}} e^{-N_{\varepsilon}^{2} /\left(2 \bar{T}_{\varepsilon} D\right)},
$$


where the second inequality follows by the reflection principle and the last inequality follows by a simple bound on Gaussian tail probabilities. By choosing $N_{\varepsilon}$ sufficiently large, we get

$$
\limsup _{\sigma \rightarrow \infty} I_{1}^{\varepsilon} \leq \varepsilon / 100 .
$$

Thus, there exists $\sigma_{\varepsilon}$, such that for all $\sigma \geq \sigma_{\varepsilon}$ we have

$$
I_{1}^{\varepsilon} \leq \varepsilon / 50 .
$$

Next, we bound $I_{2}^{\varepsilon}$. Let $\mathbb{P}^{B^{f}, B}$ be the measure induced on the canonical path space by the zeromean Brownian motions $B^{f}, B$, such that $B^{f}$ has variance $D, B$ has variance 1 , and the covariance of $B^{f}$ and $B$ is $c_{f}$, and let $\mathbb{E}^{B^{f}, B}$ be the corresponding expectation. We use again Corollary [3.6. properties of weak convergence, the dominated convergence theorem (we can switch to the Skorohod space if needed) to get

$$
\begin{aligned}
& \limsup _{\sigma \rightarrow \infty} I_{2}^{\varepsilon}=\limsup _{\sigma \rightarrow \infty} \mathbb{E}_{w}\left[\exp \left(M_{\bar{T}_{\varepsilon}}^{f, \sigma^{4}}-\frac{1}{2} A_{\bar{T}_{\varepsilon}}^{f, \sigma^{4}}\right) \mathbf{1}\left(\sup _{0 \leq t \leq \bar{T}_{\varepsilon}} R_{t}^{\sigma^{4}} \geq\left(c_{f}+\delta_{\varepsilon}\right) \bar{T}_{\varepsilon}\right) \mathbf{1}\left(\sup _{0 \leq t \leq \bar{T}_{\varepsilon}} M_{t}^{f, \sigma^{4}}<N_{\varepsilon}\right)\right] \\
& \left.\leq \mathbb{E}^{B^{f}, B}\left[e^{B_{\bar{T}_{\varepsilon}}^{f}-\frac{1}{2} D \bar{T}_{\varepsilon}}\right) \mathbf{1}\left(\sup _{0 \leq t \leq \bar{T}_{\varepsilon}} B_{t} \geq\left(c_{f}+\delta_{\varepsilon}\right) \bar{T}_{\varepsilon}\right) \mathbf{1}\left(\sup _{0 \leq t \leq \bar{T}_{\varepsilon}} B_{t}^{f} \leq N_{\varepsilon}\right)\right] \\
& \leq \mathbb{E}^{B^{f}, B}\left[e^{B_{\bar{T}_{\varepsilon}}^{f}-\frac{1}{2} D \bar{T}_{\varepsilon}} \mathbf{1}\left(\sup _{0 \leq t \leq \bar{T}_{\varepsilon}} B_{t} \geq\left(c_{f}+\delta_{\varepsilon}\right) \bar{T}_{\varepsilon}\right)\right]=\mathbb{P}^{B}\left(\sup _{0 \leq t \leq \bar{T}_{\varepsilon}}\left(B_{t}+c_{f} t\right) \geq\left(c_{f}+\delta_{\varepsilon}\right) \bar{T}_{\varepsilon}\right) .
\end{aligned}
$$

In the last equality we used the Girsanov theorem, since under the $\exp \left(B_{\bar{T}_{\varepsilon}}^{f}-\frac{1}{2} D \bar{T}_{\varepsilon}\right)$ change of measure, $B$ is a Brownian motion with the drift $2 c_{f}$ (recall that the covariance of $B^{f}$ and $B$ is $c_{f}$ ). Now it is easy to get

$$
\mathbb{P}^{B}\left(\sup _{0 \leq t \leq \bar{T}_{\varepsilon}}\left(B_{t}+c_{f} t\right) \geq\left(c_{f}+\delta_{\varepsilon}\right) \bar{T}_{\varepsilon}\right) \leq \mathbb{P}^{B}\left(\sup _{0 \leq t \leq \bar{T}_{\varepsilon}} B_{t} \geq \delta_{\varepsilon} \bar{T}_{\varepsilon}\right) \leq 2 e^{-\delta_{\varepsilon}^{2} \bar{T}_{\varepsilon} / 2} \leq \varepsilon / 100,
$$

where in the second inequality we again used reflection principle and a bound on Gaussian tail, and the last inequality follows from (4.21). Hence, there is $\sigma_{\varepsilon}$ such that for all $\sigma \geq \sigma_{\varepsilon}$, we have

$$
I_{2}^{\varepsilon} \leq \varepsilon / 50 .
$$

Combining the above estimates, we get that for $\sigma \geq \sigma_{\varepsilon}$ we have

$$
\mathbb{P}_{v}\left(G_{0}^{c}\right) \leq 2 \varepsilon / 50 \leq \varepsilon / 10,
$$

so that

$$
\mathbb{P}_{v}\left(G_{0}\right) \geq 1-\varepsilon / 10 \text {. }
$$

This finishes the proof of Lemma 4.2 .

\section{Proof of Theorem 1.2: the lower bound on the speed}

We now prove the lower bound on $V(\sigma)$.

Proposition 5.1. Suppose that $u_{0}$ satisfies (1.12) and $f$ satisfies (1.16). Then with probability 1, we have

$$
\liminf _{\sigma \rightarrow \infty} \sigma^{2} V(\sigma) \geq c_{f}
$$

The proof of Proposition 5.1 follows a similar strategy to that of Proposition 4.1. As in the proof of the upper bound, using the comparison principle and shift invariance in law, we may assume without loss of generality that $u_{0}(x)=v_{0}(x)=\mathbf{1}(x \leq 0)$. 


\subsection{Time steps for the lower bound}

We start with the definition of the time steps. The main difference with the proof of the upper bound is that we will sometimes update "backwards", and that the "good events" will be when the stopping time happens before a fixed time length rather than when the stopping times happen at a deterministic time steps, as in (4.14) in the proof of the upper bound. We will define stopping times $0=\tau_{0} \leq \tau_{1} \leq \cdots$, and a sequence $v_{t}^{(m)}(x)$ of random processes, which will be solutions to (1.14), for $t \geq \tau_{m}$, such that for each $m=0,1,2, \ldots$ the following conditions will hold almost surely:

$$
\begin{aligned}
v_{t}(x) & \geq v_{t}^{(m)}(x), \quad \text { for } t \geq \tau_{m}, x \in \mathbb{R} \\
v_{\tau_{m}}^{(m-1)}(x) & \geq v_{\tau_{m}}^{(m)}(x), \quad \text { for } x \in \mathbb{R} .
\end{aligned}
$$

Given (5.1) and (5.2), it would follow almost surely for all $m=0,1,2, \ldots$ and for all $t \geq \tau_{m}$, that

$$
L\left(v_{t}\right) \geq L\left(v_{t}^{(m)}\right)
$$

Thus, to bound $L\left(v_{t}\right)$ from below, it would suffice to bound $L\left(v_{t}^{(m)}\right)$.

We now describe the induction, starting with $\tau_{0}=0$, and $v_{t}^{(0)}(x)=v_{t}(x)$, so that (5.1) holds for $m=0$. Also define $v_{t}^{(-1)}(x)=1$, so that (5.2) holds. Let us fix some constants $\tilde{\lambda}_{1}, \widetilde{T}_{0}, N>0$, to be specified later. Suppose that we have defined $\tau_{m}$ for $0 \leq m \leq k$ and $v_{t}^{(m)}$ for $t \geq \tau_{m}$ and $0 \leq m \leq k$, and that (5.1) and (5.2) hold for $0 \leq m \leq k$. To define $\tau_{k+1}$, we consider, as in (4.12),

$$
M_{t}^{f, v, k}:=\int_{\tau_{k}}^{t} \int_{\mathbb{R}} \frac{f\left(v_{s}^{(k)}(x)\right)}{\sqrt{v_{s}^{(k)}(x)\left(1-v_{s}^{(k)}(x)\right)}} W(d x, d s),
$$

and set

$$
\tau_{k+1}=\inf \left\{t \in\left[\tau_{k}, \tau_{k}+\widetilde{T}_{0} \sigma^{8}\right]:\left|L\left(v_{t}^{(k)}\right)-L\left(v_{\tau_{k}}^{(k)}\right)\right| \geq \lambda_{1} \sigma^{4} \text { or } \frac{1}{\sigma^{4}} M_{t}^{f, v, k} \geq N\right\}
$$

with the convention $\tau_{k+1}=\tau_{k}+\widetilde{T}_{0} \sigma^{8}$ if the above set is empty.

We then let $v_{t}^{(k+1)}(x)$ for $t \geq \tau_{k+1}, x \in \mathbb{R}$ be the solution to (1.14) with the initial condition

$$
v_{\tau_{k+1}}^{(k+1)}(x)= \begin{cases}\mathbf{1}\left(x \leq L\left(v_{\tau_{k+1}}^{(k)}\right)\right), & \text { if } \tau_{k+1}<\tau_{k}+\widetilde{T}_{0} \sigma^{8} \\ \mathbf{1}\left(x \leq L\left(v_{\tau_{k}}^{(k)}\right)-\lambda_{1} \sigma^{4}\right), & \text { if } \tau_{k+1}=\tau_{k}+\widetilde{T}_{0} \sigma^{8}\end{cases}
$$

Then for $m=k+1$, the comparison principle gives (5.1), and (5.2) is true by definition.

As before, we write

$$
\Delta \tau_{k}=\tau_{k+1}-\tau_{k}
$$

and

$$
\Delta L_{k}=L\left(v_{\tau_{k+1}}^{(k)}\right)-L\left(v_{\tau_{k}}^{(k)}\right)
$$

Note that $\left\{\left(\Delta \tau_{m}, \Delta L_{k}\right)\right\}$ are i.i.d. random variables. 


\subsection{A good event and its consequences for lower bound}

We define the "good" events

$$
\widetilde{G}_{0}^{(m)}=\left\{\Delta \tau_{m}<\widetilde{T}_{0} \sigma^{8}\right\}
$$

and

$$
\begin{aligned}
& \widetilde{G}_{0}^{(1, m)}=\left\{\Delta \tau_{m}<\widetilde{T}_{0} \sigma^{8}, \Delta L_{k}=\tilde{\lambda}_{1} \sigma^{4}, \sup _{\tau_{m} \leq t \leq \tau_{m+1}} \frac{1}{\sigma^{4}} M_{t}^{f, v, k}<N\right\}, \\
& \widetilde{G}_{0}^{(2, m)}=\left\{\Delta \tau_{m}<\widetilde{T}_{0} \sigma^{8}, \Delta L_{k}=-\tilde{\lambda}_{1} \sigma^{4}, \sup _{\tau_{m} \leq t \leq \tau_{m+1}} \frac{1}{\sigma^{4}} M_{t}^{f, v, k}<N\right\}, \\
& \widetilde{G}_{0}^{(3, m)}=\left\{\Delta \tau_{m}<\widetilde{T}_{0} \sigma^{8}, \sup _{\tau_{m} \leq t \leq \tau_{m+1}} \frac{1}{\sigma^{4}} M_{t}^{f, v, k}=N\right\} .
\end{aligned}
$$

To get a lower bound on $V^{(v)}(\sigma)$ we need a lower bound on $\Delta L_{m}$ as $m \rightarrow \infty$. To this end the following lemma will be helpful.

Lemma 5.2. Let $\varepsilon \in(0,1 / 10)$ be arbitrary and $\delta_{\varepsilon}=\varepsilon / 10$. There exist $T_{\varepsilon}^{*}, N_{\varepsilon}$ and $\sigma_{\varepsilon}$ so that for all $\sigma \geq \sigma_{\varepsilon}, m \geq 0, \widetilde{T}_{0}=T_{\varepsilon}^{*}, N=N_{\varepsilon}$ and

$$
\tilde{\lambda}_{1}=\left(c_{f}-\delta_{\varepsilon}\right) T_{\varepsilon}^{*}
$$

we have

$$
\begin{aligned}
& \mathbb{P}_{v}\left(\widetilde{G}_{0}^{(1, m)}\right) \geq 1-\varepsilon / 50, \\
& \mathbb{P}_{v}\left(\widetilde{G}_{0}^{(2, m)}\right) \leq \varepsilon / 20, \\
& \mathbb{P}_{v}\left(\widetilde{G}_{0}^{(3, m)}\right) \leq \varepsilon / 50 .
\end{aligned}
$$

We postpone the proof of this lemma and first give

Proof of Proposition [5.1. Let us take $\varepsilon \in\left(0, \min \left(10^{-1}, c_{f}^{-2}\right)\right)$, and choose $T_{\varepsilon}^{*}, N_{\varepsilon}$ and $\sigma_{\varepsilon}$ as in Lemma [5.2, and consider an arbitrary $\sigma \geq \sigma_{\varepsilon}$. Lemma 5.2 implies that

$$
\mathbb{P}_{v}\left(\widetilde{G}_{0}^{(1, m)}\right)-\mathbb{P}_{v}\left(\widetilde{G}_{0}^{(2, m)}\right)-\mathbb{P}_{v}\left(\widetilde{G}_{0}^{(3, m)}\right)-\left(1-\mathbb{P}_{v}\left(\widetilde{G}_{0}^{(m)}\right)\right) \geq 1-\varepsilon / 5,
$$

for all $\sigma \geq \sigma_{\varepsilon}$, so that for all $m \geq 0$ we have

$$
\begin{aligned}
\mathbb{E}_{v}\left[\Delta L_{m}\right] & \geq \tilde{\lambda}_{1} \sigma^{4}\left(\mathbb{P}_{v}\left(\widetilde{G}_{0}^{(1, m)}\right)-\mathbb{P}_{v}\left(\widetilde{G}_{0}^{(2, m)}\right)-P_{v}\left(\widetilde{G}_{0}^{(3, m)}\right)-\left(1-P_{v}\left(\widetilde{G}_{0}^{(m)}\right)\right)\right) \\
& \geq \tilde{\lambda}_{1} \sigma^{4}(1-\varepsilon / 5) .
\end{aligned}
$$

Then using the strong law of large numbers, we have that $\mathbb{P}_{v}$ almost surely,

$$
\lim _{m \rightarrow \infty} \frac{L\left(v_{\tau_{m}}^{(m)}\right)}{m} \geq \tilde{\lambda}_{1}(1-\varepsilon / 5) \sigma^{4}
$$

for all $\sigma \geq \sigma_{\varepsilon}$. Since $\tau_{m} \leq m T_{\varepsilon}^{*} \sigma^{8}$, we have that $\mathbb{P}_{v}$ almost surely,

$$
\liminf _{m \rightarrow \infty} \frac{L\left(v_{\tau_{m}}^{(m)}\right)}{\tau_{m}}=\liminf _{m \rightarrow \infty} \frac{L\left(v_{\tau_{m}}^{(m)}\right)}{m} \frac{m}{\tau_{m}} \geq \frac{\tilde{\lambda}_{1}(1-\varepsilon / 5) \sigma^{4}}{T_{\varepsilon}^{*} \sigma^{8}}=\frac{\tilde{\lambda}_{1}(1-\varepsilon / 5)}{T_{\varepsilon}^{*}} \sigma^{-4} .
$$


Furthermore, since for $\tau_{m} \leq t \leq \tau_{m+1}$ we have

$$
L\left(v_{t}^{(m)}\right) \geq L\left(v_{\tau_{m}}^{(m)}\right)-\tilde{\lambda}_{1} \sigma^{4}
$$

and $\Delta \tau_{m} \leq T_{\varepsilon}^{*} \sigma^{8}$, it follows that, $P_{v}$ almost surely and since $\varepsilon<10^{-1}$,

$$
\begin{aligned}
V^{(v)}(\sigma) & \geq \liminf _{m \rightarrow \infty} \inf _{\tau_{m} \leq t \leq \tau_{m+1}} \frac{L\left(v_{t}^{(m)}\right)}{t} \geq \liminf _{m \rightarrow \infty} \frac{L\left(v_{\tau_{m}}^{(m)}\right)-\tilde{\lambda}_{1} \sigma^{4}}{\tau_{m}+T_{\varepsilon}^{*} \sigma^{8}} \geq \frac{\tilde{\lambda}_{1}(1-\varepsilon / 5)}{T_{\varepsilon}^{*}} \sigma^{-4} \\
& =\frac{\left(c_{f}-\varepsilon / 10\right) T_{\varepsilon}^{*}(1-\varepsilon / 5)}{T_{\varepsilon}^{*}} \sigma^{-4} \geq\left(c_{f}-\frac{\varepsilon}{5}\left(c_{f}+1\right)\right) \sigma^{-4} \\
& \geq\left(c_{f}-\sqrt{\varepsilon}\right) \sigma^{-4} .
\end{aligned}
$$

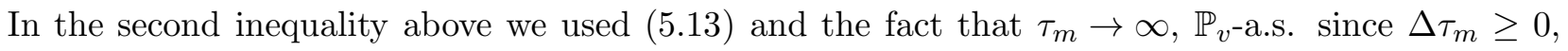
not identically zero and i.i.d. Since $\varepsilon$ was chosen to be arbitrary small we are done.

\subsection{Proof of Lemma 5.2}

As $\left(\Delta_{\tau_{m}}, \Delta L_{m}\right)$ are i.i.d., the events $G_{0}^{(m)}$ are also i.i.d., hence we only need to prove (5.8)-(5.10) for $m=0$ and write

$$
\widetilde{G}_{0}=\widetilde{G}_{0}^{(0)}, \widetilde{G}_{0}^{(i)}=\widetilde{G}_{0}^{(i, 0)}, i=1,2,3 .
$$

Fix $\varepsilon \in\left(0,10^{-1}\right)$, let $\delta_{\varepsilon}=\varepsilon / 10$, and let $T_{\varepsilon}^{*}$ be sufficiently large so that

$$
\mathbb{P}^{B}\left(B_{1} \geq-\delta_{\varepsilon} \sqrt{T}, \inf _{0 \leq t \leq 1} B_{t}>-\left(c_{f}-\delta_{\varepsilon}\right) \sqrt{T}\right) \geq 1-\varepsilon / 100, \quad \forall T \geq T_{\varepsilon}^{*} .
$$

We consider $N_{\varepsilon}>\left(2+\delta_{\varepsilon}\right) T_{\varepsilon}^{*} D$ sufficiently large, with a precise value to be specified later, and define the stopping time

$$
\xi^{\varepsilon}=\inf \left\{t \geq 0: M_{t}^{f, \sigma^{4}} \geq N_{\varepsilon}\right\} .
$$

Then by Girsanov's theorem, and since

$$
\left\{\sup _{0 \leq t \leq \Delta \tau_{0}} \frac{1}{\sigma^{4}} M_{t}^{f}<N_{\varepsilon}\right\} \supset\left\{\sup _{0 \leq t \leq T_{\varepsilon}^{*} \sigma^{8}} \frac{1}{\sigma^{4}} M_{t}^{f}<N_{\varepsilon}\right\}=\left\{\xi^{\varepsilon}>T_{\varepsilon}^{*}\right\}
$$

we have

$$
\begin{aligned}
\mathbb{P}_{v}\left(\widetilde{G}_{0}^{(1)}\right)= & \mathbb{E}_{w}\left[\exp \left(Z_{\sigma^{8}\left(T_{\varepsilon}^{*} \wedge \xi^{\varepsilon}\right)}\right) \mathbf{1}_{\widetilde{G}_{0}^{(1)}}\right] \geq E_{w}\left[\exp \left(\sigma^{-4}\left(M_{\sigma^{8}\left(T_{\varepsilon}^{*} \wedge \xi^{\varepsilon}\right)}^{f}-\frac{1}{2} \sigma^{-4} A_{\sigma^{8}\left(T_{\varepsilon}^{*} \wedge \xi^{\varepsilon}\right)}^{f}\right)\right)\right. \\
& \times \mathbf{1}\left(L(w(t)) \geq \tilde{\lambda}_{1} \sigma^{4} \text { for some } 0 \leq t \leq \sigma^{8}\left(T_{\varepsilon}^{*} \wedge \xi^{\varepsilon}\right)\right) \\
& \times \mathbf{1}\left(L(w(t))>-\tilde{\lambda}_{1} \sigma^{4} \text { for all } 0 \leq t \leq \sigma^{8}\left(T_{\varepsilon}^{*} \wedge \xi^{\varepsilon}\right)\right) \\
& \left.\times \mathbf{1}\left(\frac{1}{\sigma^{4}} M_{t}^{f}<N_{\varepsilon} \text { for all } 0 \leq t \leq \sigma^{8}\left(T_{\varepsilon}^{*} \wedge \xi^{\varepsilon}\right)\right)\right] \\
\geq & \mathbb{E}_{w}\left[\exp \left(M_{T_{\varepsilon}^{*}}^{f, \sigma^{4}}-\frac{1}{2} A_{T_{\varepsilon}^{*}}^{f, \sigma^{4}}\right) \mathbf{1}\left(L_{t}^{\sigma^{4}}>\tilde{\lambda}_{1} \text { for some } 0 \leq t \leq T_{\varepsilon}^{*}\right)\right. \\
& \left.\times \mathbf{1}\left(L_{t}^{\sigma^{4}}>-\tilde{\lambda}_{1} \text { for all } 0 \leq t \leq T_{\varepsilon}^{*}\right) \times \mathbf{1}\left(\sup _{0 \leq t \leq T_{\varepsilon}^{*}} M^{f, \sigma^{4}}<N_{\varepsilon}\right)\right]=: J_{1}^{\varepsilon} .
\end{aligned}
$$


Next, passing to the limit $\sigma \rightarrow+\infty$, we obtain, using the weak convergence in Corollary 3.6 ,

$$
\begin{aligned}
& \liminf _{\sigma \rightarrow \infty} J_{1}^{\varepsilon}=\liminf _{\sigma \rightarrow \infty} \mathbb{E}_{w} {\left[\exp \left(M_{T_{\varepsilon}^{*}}^{f, \sigma^{4}}-\frac{1}{2} A_{T_{\varepsilon}^{*}}^{f, \sigma^{4}}\right) \times \mathbf{1}\left(L_{t}^{\sigma^{4}}>\left(c_{f}-\delta_{\varepsilon}\right) T_{\varepsilon}^{*} \text { for some } 0 \leq t \leq T_{\varepsilon}^{*}\right)\right.} \\
&\left.\times \mathbf{1}\left(L_{t}^{\sigma^{4}}>-\left(c_{f}-\delta_{\varepsilon}\right) T_{\varepsilon}^{*} \text { for all } 0 \leq t \leq T_{\varepsilon}^{*}\right) \times \mathbf{1}\left(\sup _{0 \leq t \leq T_{\varepsilon}^{*}} M^{f, \sigma^{4}}<N_{\varepsilon}\right)\right] \\
& \geq \mathbb{E}^{B^{f}, B}\left[e^{B_{T_{\varepsilon}^{*}}^{f}-\frac{1}{2} D T_{\varepsilon}^{*}} \mathbf{1}\left(\sup _{0 \leq t \leq T_{\varepsilon}^{*}} B_{t}>\left(c_{f}-\delta_{\varepsilon}\right) T_{\varepsilon}^{*}\right) \mathbf{1}\left(\inf _{0 \leq t \leq T_{\varepsilon}^{*}} B_{t}>-\left(c_{f}-\delta_{\varepsilon}\right) T_{\varepsilon}^{*}\right)\right. \\
&\left.\times \mathbf{1}\left(\sup _{0 \leq t \leq T_{\varepsilon}^{*}} B_{t}^{f}<N_{\varepsilon}\right)\right] .
\end{aligned}
$$

We rewrite this, using Girsanov's theorem for correlated Brownian motions with a drift, as

$$
\begin{aligned}
\liminf _{\sigma \rightarrow \infty} J_{1}^{\varepsilon} \geq & \mathbb{E}^{B^{f}, B}\left[e^{B_{T_{\varepsilon}^{*}}^{f}-\frac{1}{2} D T_{\varepsilon}^{*}} \mathbf{1}\left(\sup _{0 \leq t \leq T_{\varepsilon}^{*}} B_{t}>\left(c_{f}-\delta_{\varepsilon}\right) T_{\varepsilon}^{*}\right) \times \mathbf{1}\left(\inf _{0 \leq t \leq T_{\varepsilon}^{*}} B_{t}>-\left(c_{f}-\delta_{\varepsilon}\right) T_{\varepsilon}^{*}\right)\right] \\
& -\mathbb{E}^{B^{f}, B}\left[e^{B_{T_{\varepsilon}^{*}}^{f}-\frac{1}{2} D T_{\varepsilon}^{*}} \mathbf{1}\left(\sup _{0 \leq t \leq T_{\varepsilon}^{*}} B_{t}^{f} \geq N_{\varepsilon}\right)\right] \\
= & \mathbb{P}^{B}\left(\sup _{0 \leq t \leq T_{\varepsilon}^{*}}\left(B_{t}+c_{f} t\right) \geq\left(c_{f}-\delta_{\varepsilon}\right) T_{\varepsilon}^{*}, \inf _{0 \leq t \leq T_{\varepsilon}^{*}}\left(B_{t}+c_{f} t\right)>-\left(c_{f}-\delta_{\varepsilon}\right) T_{\varepsilon}^{*}\right) \\
& -\mathbb{P}^{B^{f}}\left(\sup _{0 \leq t \leq T_{\varepsilon}^{*}} B_{t}^{f}+D t \geq N_{\varepsilon}\right) .
\end{aligned}
$$

The first term in the right side can be bounded as

$$
\begin{aligned}
\mathbb{P}^{B} & \left(\sup _{0 \leq t \leq T_{\varepsilon}^{*}}\left(B_{t}+c_{f} t\right) \geq\left(c_{f}-\delta_{\varepsilon}\right) T_{\varepsilon}^{*}, \inf _{0 \leq t \leq T_{\varepsilon}^{*}}\left(B_{t}+c_{f} t\right)>-\left(c_{f}-\delta_{\varepsilon}\right) T_{\varepsilon}^{*}\right) \\
& \geq \mathbb{P}^{B}\left(B_{T_{\varepsilon}^{*}}+c_{f} T_{\varepsilon}^{*} \geq\left(c_{f}-\delta_{\varepsilon}\right) T_{\varepsilon}^{*}, \inf _{0 \leq t \leq T_{\varepsilon}^{*}} B_{t}>-\left(c_{f}-\delta_{\varepsilon}\right) T_{\varepsilon}^{*}\right) \\
& =\mathbb{P}^{B}\left(B_{T_{\varepsilon}^{*}} \geq-\delta_{\varepsilon} T_{\varepsilon}^{*}, \inf _{0 \leq t \leq T_{\varepsilon}^{*}} B_{t}>-\left(c_{f}-\delta_{\varepsilon}\right) T_{\varepsilon}^{*}\right) \\
& =\mathbb{P}^{B}\left(B_{1} \geq-\delta_{\varepsilon} \sqrt{T_{\varepsilon}^{*}}, \inf _{0 \leq t \leq 1} B_{t}>-\left(c_{f}-\delta_{\varepsilon}\right) \sqrt{T_{\varepsilon}^{*}}\right) \geq 1-\varepsilon / 100
\end{aligned}
$$

where the last inequality follows by (5.14). The second term in the right side of (5.17) can be bounded using the reflection principle for Brownian motion, bounds on tails of Gaussian probabilities and by choosing $N_{\varepsilon} \geq\left(2+\delta_{\varepsilon}\right) T_{\varepsilon}^{*} D$ sufficiently large, so that

$$
\begin{aligned}
\mathbb{P}^{B^{f}}\left(\sup _{0 \leq t \leq T_{\varepsilon}^{*}} B_{t}^{f}+D t \geq N_{\varepsilon}\right) \leq \mathbb{P}^{B^{f}}\left(\sup _{0 \leq t \leq T_{\varepsilon}^{*}} B_{t}^{f} \geq N_{\varepsilon}-D T_{\varepsilon}^{*}\right) \\
\quad \leq 2 \mathbb{P}^{B}\left(\sqrt{D} B_{T_{\varepsilon}^{*}} \geq N_{\varepsilon}-D T_{\varepsilon}^{*}\right) \leq 2 \exp \left(-\frac{\left(N_{\varepsilon}-D T_{\varepsilon}^{*}\right)^{2}}{2 T_{\varepsilon}^{*} D}\right) \leq \varepsilon / 100 .
\end{aligned}
$$

Combining (5.15)-(5.19) we get that for $N_{\varepsilon}$ sufficiently large we have

$$
\mathbb{P}_{v}\left(\widetilde{G}_{0}^{(1)}\right) \geq 1-\varepsilon / 50
$$

which is (5.8). 
Next, we bound $\mathbb{P}_{v}\left(\widetilde{G}_{0}^{(2)}\right)$. Again, using Girsanov's theorem we write

$$
\begin{aligned}
& \mathbb{P}_{v}\left(\widetilde{G}_{0}^{(2)}\right)=\mathbb{E}_{w}\left[\exp \left(Z_{\sigma^{8}\left(T_{\varepsilon}^{*} \wedge \xi^{\varepsilon}\right)}\right) \mathbf{1}_{\widetilde{G}_{0}^{(2)}}\right] \leq \mathbb{E}_{w}\left[\operatorname { e x p } \left(\sigma^{-4}\left(M_{\sigma^{8}\left(T_{\varepsilon}^{*} \wedge \xi^{\varepsilon}\right)}^{f}-\frac{1}{2} \sigma^{-4} A_{\sigma^{8}\left(T_{\varepsilon}^{*} \wedge \xi^{\varepsilon}\right)}^{f}\right)\right.\right. \\
& \quad \times \mathbf{1}\left(L\left(w_{t}\right) \leq-\tilde{\lambda}_{1} \sigma^{4} \text { for some } 0 \leq t \leq \sigma^{8}\left(T_{\varepsilon}^{*} \wedge \xi^{\varepsilon}\right)\right) \\
& \left.\quad \times \mathbf{1}\left(\frac{1}{\sigma^{4}} M_{t}^{f}<N_{\varepsilon} \text { for all } 0 \leq t \leq \sigma^{8} T_{\varepsilon}^{*}\right)\right] \\
& \quad+\mathbb{E}_{w}\left[\exp \left(\sigma^{-4}\left(M_{\sigma^{8}\left(T_{\varepsilon}^{*} \wedge \xi^{\varepsilon}\right)}^{f}-\frac{1}{2} \sigma^{-4} A_{\sigma^{8}\left(T_{\varepsilon}^{*} \wedge \xi^{\varepsilon}\right)}^{f}\right)\right) \times \mathbf{1}\left(\sup _{0 \leq t \leq \sigma^{8} T_{\varepsilon}^{*}} \frac{1}{\sigma^{4}} M_{t}^{f} \geq N_{\varepsilon}\right)\right] \\
& \leq \mathbb{E}_{w}\left[\exp \left(M_{T_{\varepsilon}^{*}}^{f, \sigma^{4}}-\frac{1}{2} A_{T_{\varepsilon}^{*}}^{f, \sigma^{4}}\right) \times \mathbf{1}\left(\inf _{0 \leq t \leq T_{\varepsilon}^{*}} L_{t}^{\sigma^{4}} \leq-\tilde{\lambda}_{1}\right) \times \mathbf{1}\left(\sup _{0 \leq t \leq T_{\varepsilon}^{*}} M^{f, \sigma^{4}}<N_{\varepsilon}\right)\right] \\
& \quad+\mathbb{E}_{w}\left[\exp \left(\sigma^{-4}\left(M_{\sigma^{8}\left(T_{\varepsilon}^{*} \wedge \xi^{\varepsilon}\right)}^{f}-\frac{1}{2} \sigma^{-4} A_{\sigma^{8}\left(T_{\varepsilon}^{*} \wedge \xi^{\varepsilon}\right)}^{f}\right) \times \mathbf{1}\left(\sup _{0 \leq t \leq \sigma^{8} T_{\varepsilon}^{*}} \frac{1}{\sigma^{4}} M_{t}^{f} \geq N_{\varepsilon}\right)\right]=: J_{2,1}^{\varepsilon}+J_{2,2}^{\varepsilon} .\right.
\end{aligned}
$$

The term $J_{2,2}^{\varepsilon}$ is exactly as $I_{1}^{\varepsilon}$ in (4.23), thus, as in (4.25) we have, by choosing $N_{\varepsilon}$ sufficiently large:

$$
J_{2,2}^{\varepsilon} \leq \varepsilon / 50
$$

for all $\sigma$ sufficiently large. As for $J_{2,1}^{\varepsilon}$, proceeding similarly to (5.16), we obtain

$$
\begin{aligned}
\limsup _{\sigma \rightarrow \infty} J_{2,1}^{\varepsilon} & \leq \mathbb{E}^{B^{f}, B}\left[e^{B_{T_{\varepsilon}^{*}}^{f}-\frac{1}{2} D T_{\varepsilon}^{*}} \mathbf{1}\left(\inf _{0 \leq t \leq T_{\varepsilon}^{*}} B_{t} \leq-\left(c_{f}-\delta_{\varepsilon}\right) T_{\varepsilon}^{*}\right) \times \mathbf{1}\left(\sup _{0 \leq t \leq T_{\varepsilon}^{*}} B_{t}^{f} \leq N_{\varepsilon}\right)\right] \\
& \leq \mathbb{P}^{B}\left(\inf _{0 \leq t \leq T_{\varepsilon}^{*}}\left(B_{t}+c_{f} t\right) \leq-\left(c_{f}-\delta_{\varepsilon}\right) T_{\varepsilon}^{*}\right) \leq \mathbb{P}^{B}\left(\inf _{0 \leq t \leq 1} B_{t} \leq-\left(c_{f}-\delta_{\varepsilon}\right) \sqrt{T_{\varepsilon}^{*}}\right) \\
& \leq \varepsilon / 100 .
\end{aligned}
$$

Here, the last inequality follows from (5.14). Combining (5.21) and (5.22) we see that for $N_{\varepsilon}$ sufficiently large we have

$$
\liminf _{\sigma \rightarrow \infty} J_{2}^{\varepsilon} \leq 3 \varepsilon / 100
$$

and (5.9) follows.

To bound $\widetilde{G}_{0}^{(3)}$, once again by Girsanov's theorem and recalling the definition of $J_{2,2}^{\varepsilon}$, we obtain

$$
\begin{aligned}
\mathbb{P}_{v}\left(\widetilde{G}_{0}^{(3)}\right)= & \mathbb{E}_{w}\left[\exp \left(Z_{\sigma^{8}\left(T_{\varepsilon}^{*} \wedge \xi^{\varepsilon}\right)}\right) \mathbf{1}_{\widetilde{G}_{0}^{(3)}}\right] \leq \mathbb{E}_{w}\left[\exp \left(\sigma^{-4}\left(M_{\sigma^{8}\left(T_{\varepsilon}^{*} \wedge \xi^{\varepsilon}\right)}^{f}-\frac{1}{2} \sigma^{-4} A_{\sigma^{8}\left(T_{\varepsilon}^{*} \wedge \xi^{\varepsilon}\right)}^{f}\right)\right)\right. \\
& \left.\times \mathbf{1}\left(\sup _{0 \leq t \leq T_{\varepsilon}^{*}} M^{f, \sigma^{4}} \geq N_{\varepsilon}\right)\right]=J_{2,2}^{\varepsilon} \leq \varepsilon / 50,
\end{aligned}
$$

where the last inequality follows from (5.21) for $N_{\varepsilon}$ sufficiently large and all $\sigma$ sufficiently large. Thus (5.10) follows, and the proof of Lemma 5.2 is complete.

\section{References}

[AE86] J. Aguirre and M. Escobedo, A Cauchy problem for $u_{t}-\Delta u=u^{p}$ with $0<p<1$. Asymptotic behaviour of solutions. Ann. Fac. Sci. Toulouse Math. (5), 8(2):175-203, 1986/87.

[AW78] D. Aronson, and H. Weinberger, Multidimensional nonlinear diffusion arising in population genetics, Adv. in Math. 30 (1978), 33-76. 
[Bra78] M.D. Bramson, Maximal displacement of branching Brownian motion, Comm. Pure Appl. Math. 31, 1978, 531-581.

[Bra83] M.D. Bramson, Convergence of solutions of the Kolmogorov equation to travelling waves, Mem. Amer. Math. Soc. 44, 1983.

[BD97] Éric Brunet and Bernard Derrida, Shift in the velocity of a front due to a cutoff, Phys. Rev. E (3) 56 (1997), no. 3, part A, 2597-2604.

[BD00] Éric Brunet and Bernard Derrida, Probability distribution of the free energy of a directed polymer in a random medium, Phys. Rev. E (3) 61 (2000), no. 6, part B, 6789-6801.

[CD05] Joseph G. Conlon and Charles R. Doering, On travelling waves for the stochastic FisherKolmogorov-Petrovsky-Piscunov equation, J. Stat. Phys. 120 (2005), no. 3-4, 421-477.

[Daw78] D.A. Dawson, Geostochastic calculus, Canadian J. Statistics 6 (1978), 143-168.

[Fis37] R.A. Fisher, The wave of advance of advantageous genes., Annals of Eugenics 7 (1937), $355-369$.

[MMQ11] Carl Mueller, Leonid Mytnik, and Jeremy Quastel, Effect of noise on front propagation in reaction-diffusion equations of kpp type, Invent. Math. 184 (2011), no. 2, 405-453.

[MS95] C. Mueller and R. Sowers, Random traveling waves for the KPP equation with noise, J. Funct. Anal. 128 (1995), 439-498.

[MT97] C. Mueller and R. Tribe, Finite width for a random stationary interface, Electronic J. Prob. 2 (1997), 1-27, Paper no. 7.

[Mue91] C. Mueller, On the support of solutions to the heat equation with noise, Stochastics 37 (1991), no. 4, 225-246.

[NRR18] J. Nolen, J.-M. Roquejoffre and L. Ryzhik, Refined long time asymptotics for Fisher-KPP fronts, Preprint, 2018.

[Per02] E. Perkins, Dawson-Watanabe superprocesses and measure-valued diffusions. Lectures on probability theory and statistics (Saint-Flour, 1999), 125-324, Lecture Notes in Math., 1781, Springer, Berlin, 2002.

[Rob13] M. Roberts, A simple path to asymptotics for the frontier of a branching Brownian motion, Ann. Prob. 41, 2013, 3518-3541.

[Shi88] T. Shiga, Stepping stone models in population genetics and population dynamics. Stochastic processes in physics and engineering (Bielefeld, 1986), 345-355, Math. Appl., 42, Reidel, Dordrecht, 1988.

[Shi94] T. Shiga, Two contrasting properties of solutions for one-dimensional stochastic partial differential equations, Can. J. Math 46 (1994), no. 2, 415-437.

[Tri95] R. Tribe, Large time behavior of interface solutions to the heat equation with Fisher-Wright noise, Probab. Theory Related Fields 102 (1995), 289-311.

[Wal86] J.B. Walsh, An introduction to stochastic partial differential equations, École d'été de probabilités de Saint-Flour, XIV-1984 (Berlin, Heidelberg, New York) (P. L. Hennequin, ed.), Lecture Notes in Mathematics 1180, Springer-Verlag, 1986, pp. 265-439. 